\title{
REVEALED STOCHASTIC PREFERENCE: A SYNTHESIS ${ }^{1}$
}

\author{
Daniel L. McFadden* \\ * Department of Economics, University of California, Berkeley CA 94720-3880 USA \\ (e-mail: mcfadden@econ.berkeley.edu)
}

Submitted: December 14, 2002; revised version February 10, 2004

Summary: The problem of revealed stochastic preference is whether probability distributions of observed choices in a population for various choice situations are consistent with a hypothesis of maximization of preference preorders by members of the population. This is a population analog of the classical revealed preference problem in economic consumer theory. This paper synthesizes the solutions to this problem that have been obtained by Marcel K. Richter and the author, and by J. C. Falmagne, in the case of finite sets of alternatives, and utilizes unpublished research of Richter and the author to give results for the non-finite choice sets encountered in economic consumer theory.

Keywords and phrases: choice, stochastic preference, revealed preference, random utility maximization

JEL Classification numbers: D1, C6

\footnotetext{
${ }^{1}$ The preparation of this paper was supported by the E. Morris Cox endowment at the University of California, Berkeley. I am indebted to Robert Anderson, Salvador Barbara, Werner Hildenbrand, Rosa L. Matzkin, and Aviv Nevo for useful suggestions and comments. I am especially indebted to Marcel K. Richter, who was the source of many of the ideas and arguments contained in this paper. Correspondence should be directed to the Department of Economics, Evans Hall, University of California, Berkeley CA 94720-3880, or mcfadden@econ.berkeley.edu.
} 


\section{Introduction}

The problem of revealed stochastic preference asks the question: Are the distributions of choices observed for a population of individuals in a variety of choice situations consistent with rational choice theory, which postulates that individuals maximize preferences? In economic consumer theory, each choice situation is defined by a budget set; in psychometrics, by the alternatives offered in an experiment; and in political voting behavior, by the issues presented in an election. Distributions of responses arise because of taste heterogeneity in the population, or because of stochastic elements in individual preferences. The last possibility connects rational choice theory to psychometric models of choice based on random scale maximization. This paper synthesizes the relatively complete solutions to the revealed preference problem that have been obtained for finite choice sets, and extends these results to the non-finite choice sets commonly encountered in economic consumer behavior. This paper is based primarily on unpublished research that Marcel K. Richter and I did in 1971, and on subsequent published results for the finite case by Falmagne (1978) and by McFadden and Richter (1990). Ket Richter has had an impact on economic theory far beyond the papers published over his name. It is a fitting tribute to his career to draw upon his unpublished ideas and words to suggest the scope and significance of his influence.

The origin of the revealed stochastic preference problem is the classical economic theory of revealed preference, where the Samuelson-Houthaker Strong Axiom of Revealed Preference (SARP) and Richter's Congruence Axiom provide tight necessary and sufficient conditions for consistency of one individual's choices with preference maximization; see Samuelson (1938), Houthaker (1950), Richter (1966,1971). Marschak (1960) connected this theory to the psychometric literature (Thurstone (1927), Luce (1959)), posing the question of when observed choice probabilities could be rationalized as consistent with random utility maximization (RUM). Papers addressing the revealed stochastic preference problem include Block and Marschak (1960), McFadden and Richter (1971,1990), McFadden (1973,1975), Falmagne (1978), Fishbern (1978), Cohen (1980), Barbara and Pattanaik (1986), McLennan (1990), 
Fishburn and Falmagne (1989), Barbara (1990), Cohen and Falmagne (1990), Fishburn (1992), and Bandyopadhyay, Dasgupta, and Pattanaik (1999). ${ }^{2}$

The ingredients of a revealed preference problem are the universe of objects of choice, a family of feasible budget sets giving the alternatives from which a decision-maker must choose, a class of permissible decision rules consistent with a specified theory of choice behavior, and observations on the probabilities of choices made. Both the SARP and the Congruence Axiom consider classes of decision rules that maximize a preference preorder. They differ in that the SARP requires permissible decision-rules to produce unique maxima on feasible budget sets, and assumes a unique offer is observed, while the Congruence Axiom allows decision rules that yield multiple maxima, and assumes that decision-makers offer the sets of acceptable alternatives in the case of ties. One can generate a variety of revealed preference problems by varying the ingredients, particularly the family of feasible sets, the class of permissible decision rules, and the structure of observations. Some of the possibilities are discussed in the conclusion.

This paper is organized as follows. Section 2 sets notation and gives a formal statement of the revealed stochastic preference problem. Section 3 reviews the revelation problem when the universe of alternatives is finite, and relates the necessary and sufficient conditions for this problem obtained by McFadden and Richter $(1971,1990)$ and by Falmagne (1978). Section 4 gives the McFadden and Richter (1971) results on the extension of set functions, together with new results on countable additivity. Section 5 draws upon this mathematical theory to obtain necessary and sufficient conditions for the revealed stochastic preference problem with a non-finite universe of alternatives that includes the classical economic consumer problem. Section 6 concludes with discussion of further extensions and problems.

\footnotetext{
${ }^{2}$ There is a large literature in mathematical psychology dealing with concepts of stochastic transitivity, and their relationship to the RUM hypothesis; see Fishburn (1999). There is also a very extensive literature on the Luce Choice Axiom (Luce, 1959), which provided the foundation for the econometric theory of discrete choice behavior; see McFadden (1974), Halldin (1974), Manski (1977), McFadden (1981), McCausland (2002). McFadden (2001) surveys this subject and provides many references.
} 


\section{The Revealed Stochastic Preference Problem}

2.1. Notation. The following notation for the space of alternatives, the choice situations, observed behavior, and the hypothesis of rational behavior will be used throughout the paper:

$(\mathbf{X}, \mathfrak{X})$ a metric space $\mathbf{X}$ of possible objects of choice, and the Borel $\sigma$-algebra $\mathfrak{X}$ of subsets of $\mathbf{X}$.

Q a non-empty index set, a metric space interpreted as indexing the feasible choice situations.

$\mathbf{B}(\mathrm{q})$ a non-empty set in $\mathfrak{X}$ for $\mathrm{q} \in \mathbf{Q}$, interpreted as the set of available alternatives, or "budget set", in choice situation q.

$\mathbf{d}: \mathbf{Q} \rightarrow \mathfrak{X} \quad$ a decision rule that maps $\mathbf{Q}$ into subsets of $\mathbf{X}$, with $\varnothing \neq \mathbf{d}(\mathrm{q}) \subseteq \mathbf{B}(\mathrm{q})$, interpreted as a behavior rule that designates the decision-maker's acceptable alternatives in $\mathbf{B}(q)$. The decision rule is decisive if $\mathbf{d}(\mathbf{q})$ is a singleton; a non-decisive choice is interpreted as the offer of a set of "tied" alternatives.

(q,C) a pair, termed a trial; composed of a feasible choice situation $\mathrm{q} \in \mathbf{Q}$ and a set $\mathbf{C} \in \mathfrak{X}$. The outcome of a trial is a success (failure) if $\mathbf{C}$ contains (excludes) the choice $\mathbf{d}(\mathbf{q})$ made by an individual in situation q. A trial can be a partial success if the decision rule is non-decisive and $\mathbf{d}(\mathbf{q})$ intersects $\mathbf{C}$ but is not contained in $\mathbf{C}$.

(D.D,$\zeta$ ) a probability space consisting of a set $\mathbf{D}$ of decision rules, a Boolean $\sigma$-algebra $\mathscr{D}$ of measurable subsets of $\mathbf{D}$, and a probability $\zeta$ on $\mathscr{D}$. This is interpreted as the universe of decision rules that could appear in a population of decision-makers.

$\Pi_{\mathrm{q}} \quad$ a choice probability on $\mathscr{X}$ for $\mathrm{q} \in \mathbf{Q}$, with $\Pi_{\mathrm{q}}(\mathbf{C})$ for $\mathbf{C} \in \mathfrak{X}$ interpreted as the proportion of individuals in the population with choice functions satisfying $\mathbf{d}(q) \subseteq \mathbf{C}$. The algebra $\mathscr{D}$ contains the sets $\mathbf{D}(\mathrm{q}, \mathbf{C})=\{\mathbf{d} \in \mathbf{D} \mid \mathbf{d}(\mathrm{q}) \subseteq \mathbf{C}\}$ for $\mathrm{q} \in \mathbf{Q}$ and $\mathbf{C} \in \mathfrak{X}$, so that the probability $\Pi_{\mathrm{q}}(\mathbf{C}) \equiv \zeta(\mathbf{D}(\mathrm{q}, \mathbf{C}))$ that the trial $(\mathrm{q}, \mathbf{C})$ is a success is defined. The choice probability satisfies $\Pi_{\mathrm{q}}(\mathbf{B}(\mathrm{q}))=1$, and if the decision rules of the population are almost surely decisive, it satisfies $\Pi_{\mathrm{q}}(\mathbf{C})+\Pi_{\mathrm{q}}\left(\mathbf{C}^{\mathrm{c}}\right)=1$. More generally, let $\Pi_{\mathrm{q}}^{-}$and $\Pi_{\mathrm{q}}^{+}$denote set-valued bounds for $\mathrm{q} \in \mathbf{Q}$, satisfying $\Pi_{\mathrm{q}}^{-}(\mathbf{C}) \leq \zeta(\mathbf{D}(\mathrm{q}, \mathbf{C})) \leq \Pi_{\mathrm{q}}^{+}(\mathbf{C})$ for $\mathbf{C} \in \mathfrak{X}$.

$\mathbf{t}=\left\langle\left(\mathrm{q}_{1}, \mathbf{C}_{1}\right), \ldots,\left(\mathrm{q}_{\mathrm{m}}, \mathbf{C}_{\mathrm{m}}\right)>\right.$ a trial sequence, an ordered sequence with repetitions permitted, and elements $\left(\mathrm{q}_{\mathrm{i}}, \mathbf{C}_{\mathrm{i}}\right) \in \mathbf{Q} \times \mathfrak{X}$ for $\mathrm{i}=1, \ldots, \mathrm{m}$, where $\mathrm{m}$ is a positive integer. 
H a set of choice functions in $\mathbf{D}$, interpreted as the choice functions consistent with a specified hypothesis of rational choice behavior. The algebra $\mathscr{D}$ contains $\mathbf{H}$, so that the sets $\mathbf{H}(\mathrm{q}, \mathbf{C})$ $=\mathbf{D}(\mathrm{q}, \mathbf{C}) \cap \mathbf{H}$ are contained in the Boolean $\sigma$-algebra $\mathscr{H}=\{\mathbf{A} \cap \mathbf{H} \mid \mathbf{A} \in \mathcal{D}\}$ for $\mathrm{q} \in \mathbf{Q}$ and $\mathbf{C} \in$ $\mathfrak{X}$, and $\zeta(\mathbf{H}(\mathrm{q}, \mathbf{C}))$ is defined as the probability that the trial $(\mathrm{q}, \mathbf{C})$ is a success for decision rules that satisfy the rational choice hypothesis.

$\alpha_{\mathbf{H}}(\mathrm{t}) \quad$ the H-intersection number of a trial sequence $\mathbf{t}=\left\langle\left(\mathrm{q}_{1}, \mathbf{C}_{1}\right), \ldots,\left(\mathrm{q}_{\mathrm{m}}, \mathbf{C}_{\mathrm{m}}\right)\right\rangle$, defined to be the maximum number of successes for the sequence attainable by a choice function in $\mathbf{H}$ :

$$
\alpha_{\mathrm{H}}(\mathrm{t})=\max _{\mathrm{d} \in \mathbf{H}} \sum_{i=1}^{m} \quad \mathbf{1}\left(\mathbf{d}\left(\mathrm{q}_{\mathrm{i}}\right) \subseteq \mathbf{C}_{\mathrm{i}}\right) .
$$

$\mathrm{u}: \mathbf{X} \rightarrow \mathbb{R} \quad$ a utility or scale function on $\mathbf{X}$, a representation of a preference preorder. A utility function $\mathrm{u}$ is weakly decisive if $\mathbf{d}(\mathrm{q})=\mathbf{d}(\mathrm{q} ; \mathrm{u}) \equiv\left\{\mathrm{x} \in \mathbf{B}(\mathrm{q}) \mid \mathrm{u}(\mathrm{x}) \geq \mathrm{u}\left(\mathrm{x}^{\prime}\right)\right.$ for all $\left.\mathrm{x}^{\prime} \in \mathbf{B}(\mathrm{q})\right\}$ is non-empty for $\mathrm{q} \in \mathbf{Q}$, and is decisive if $\mathbf{d}(\mathrm{q} ; \mathrm{u})$ is a singleton for $\mathrm{q} \in \mathbf{Q}$.

$(\mathbf{U}, \mathcal{Q}, v)$ a non-empty set of utility functions $\mathrm{u}$ specified by a hypothesis of rational choice behavior, a metric space, with $\mathcal{U}$ the Borel $\sigma$-algebra of subsets of $\mathbf{U}$, and $v$ a probability on $\mathcal{U}$, termed a random utility maximization (RUM) model. A space of decision rules $(\mathbf{H}, \mathscr{H}, \zeta)$ and a space of utility functions $(\mathbf{U}, \mathcal{Q}, v)$ are consistent (or, the set $\mathbf{H}$ of decision

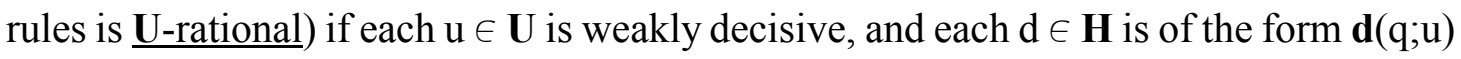
for some $\mathbf{u} \in \mathbf{U}$ and all $\mathbf{q} \in \mathbf{Q}$, the inverse image of $\mathbf{H}(\mathrm{q}, \mathbf{C})$ is in $\mathcal{U}$ for $\mathrm{q} \in \mathbf{Q}, \mathbf{C} \in \mathfrak{X}$ (i.e., $\mathbf{U}(\mathrm{q}, \mathbf{C}) \equiv\{\mathrm{u} \in \mathbf{U} \mid \mathbf{d}(\mathrm{q} ; \mathrm{u}) \subseteq \mathbf{C}$ for $\mathrm{q} \in \mathbf{Q}$ and $\mathbf{C} \in \mathfrak{X}\} \in \mathbf{U})$, and $\zeta(\mathbf{H}(\mathrm{q}, \mathbf{C}))=v(\mathbf{U}(\mathrm{q}, \mathbf{C}))$.

$\alpha_{U}(t) \quad$ the $U$-intersection number of a trial sequence $\mathbf{t}=\left\langle\left(\mathrm{q}_{1}, \mathbf{C}_{1}\right), \ldots,\left(\mathrm{q}_{\mathrm{m}}, \mathbf{C}_{\mathrm{m}}\right)>\right.$, defined to be the maximum number of successes for the sequence attainable by a utility function in $\mathbf{U}$; i.e., $\alpha_{\mathrm{U}}(\mathrm{t})=\max _{\mathrm{u} \in \mathrm{U}} \sum_{i=1}^{m} \quad \mathbf{1}\left(\mathbf{d}\left(\mathrm{q}_{\mathrm{i}}, \mathrm{u}\right) \subseteq \mathbf{C}_{\mathrm{i}}\right)$. If the space $\mathbf{H}$ of decision rules and the space $\mathbf{U}$ of utility functions are consistent, then the decision-rule and utility intersection numbers are the same.

2.2. Discussion. The central results in this paper concern random utility maximization, and utilize spaces $(\mathbf{U}, \mathcal{Q}, \boldsymbol{v})$ of weakly decisive utility functions. These results will have equivalent restatements in terms of the consistent space of decision rules $(\mathbf{H}, \mathscr{H}, \zeta)$. We will also give some results directly for a space of hypothesized decision rules $(\mathbf{H}, \mathscr{H}, \zeta)$; these can be applied to theories of choice other than random utility maximization. The universe $(\mathbf{D}, \mathscr{D}, \zeta)$ of decision rules will play no direct role in our 
analysis; but is useful in interpreting revealed preference problems as null hypotheses $\mathbf{H}$ on this universe. In this interpretation, the revealed preference problem can be viewed as an extreme case of the econometric problem of estimating the probability measure $\zeta$ or bounding $\zeta(\mathbf{H})$.

In the classical theory of economic consumer demand, each alternative is a commodity vector represented by a point in a closed consumption set $\mathbf{X}$ contained in the non-negative orthant of $\mathbb{R}^{\mathrm{n}} ; \mathbf{X}$ is often assumed to be convex. The space of choice possibilities $\mathbf{Q}$ is a set of $n$-vectors of positive commodity prices $\mathrm{q}=\left(\mathrm{q}_{1}, \ldots, \mathrm{q}_{\mathrm{n}}\right)$, where income is normalized to one. Then, the possible choice sets are the budget sets $\mathbf{B}(\mathrm{q})=\left\{\mathrm{x}=\left(\mathrm{x}_{1}, \ldots, \mathrm{x}_{\mathrm{n}}\right) \in \mathbf{X} \mid \mathrm{q}_{1} \mathrm{x}_{1}+\ldots+\mathrm{q}_{\mathrm{n}} \mathrm{x}_{\mathrm{n}} \leq 1\right\}$; with $\mathbf{Q}$ restricted so that $\mathbf{B}(\mathrm{q})$ is always non-empty. The admissible behavior rules $\mathbf{H} \subseteq \mathbf{D}$ under the theory of utility-maximizing choice behavior are those consistent with a specified family of weakly decisive utility functions $\mathbf{U}$. For this setup, it will often be natural to impose some combination of the following assumptions: [A1]. $\mathbf{X}$ is compact and convex; [A2] The feasible choice sets $\mathbf{B}(\mathrm{q})$ are closed and convex for $\mathrm{q} \in \mathbf{Q}$; [A3] $\mathbf{Q}$ is a metric space, and the mapping $\mathbf{B}(\mathbf{q})$ from $\mathbf{Q}$ into non-empty subsets of $\mathbf{X}$ is a continuous, compact-valued, convex-valued correspondence, ${ }^{3}$ [A4a] Utility functions $\mathrm{u} \in \mathbf{U}$ are uniformly bounded, continuous and quasi-concave, or uniformly Lipschitz, and strictly quasi-concave, [A4b] Utility functions $\mathrm{u} \in \mathbf{U}$ are defined on an open neighborhood of $\mathrm{X}$, and are uniformly bounded and concave.

A complete theory of choice behavior requires either (1) that the structure of the choice problem is such that decision rules are always decisive, if necessary through the introduction of explicit tie-breaking mechanisms, or (2) that decision-makers are observed to offer sets of "tied" acceptable alternatives and they passively accept assignments from their offers. An incomplete theory that does not specify tiebreaking mechanisms may nevertheless be empirically complete if in practice decision-rules are almost surely decisive. Shape restrictions may ensure that economic consumer choice is decisive; i.e., if budget sets are compact and convex, and admissible utility functions are continuous and strictly quasi-concave, then decision rules always yield singletons. However, more generally utility maximization does not rule out ties. We will assume that offer sets of admissible alternatives are observed, and define $\Pi_{\mathrm{q}}(\mathbf{C})$ to be

\footnotetext{
${ }^{3}$ A correspondence is continuous if it is upper and lower hemicontinuous in the terminology of Hildenbrand (1974, I.B.III). When the space of closed non-empty subsets of $(\mathbf{X}, \mathfrak{X})$ is metrized by the Hausdorff distance, then an equivalent characterization is that $\mathbf{B}(\mathrm{q})$ is a continuous function from $\mathbf{Q}$ into this metric space.
} 
the probability that the observed offer set in choice situation $\mathrm{q}$ is contained in $\mathbf{C}$. The sum of the probabilities $\Pi_{q}(\mathbf{C})+\Pi_{q}\left(\mathbf{C}^{c}\right)$ is less than one if the probability of a partial success (where $\mathbf{d}(q)$ intersects but is not contained in $\mathbf{C}$ ) is positive. In this case, we can consider observed lower bounds $\Pi_{q}^{-}$on the probabilities of success and upper bounds $\Pi_{\mathrm{q}}^{+}$on the probabilities of success or partial success (i.e., $\left.\Pi_{\mathrm{q}}^{+}(\mathbf{C})=1-\Pi_{\mathrm{q}}^{-}\left(\mathbf{C}^{\mathrm{c}}\right)\right)$. Alternately, if we observe $\Pi_{\mathrm{q}}(\mathbf{C})+\Pi_{\mathrm{q}}\left(\mathbf{C}^{\mathrm{c}}\right)=1$ for all $\mathbf{C} \in \mathbf{X}$, then admissible decision rules are almost surely decisive at each $\mathrm{q} \in \mathbf{Q}$, and $\Pi_{\mathrm{q}}$ is an almost surely complete description of the distribution of demand.

2.3. Revelation Problems. We define the revelation problems we will consider.

2.3.1. The Revealed Distribution Problem: If $\Pi_{q}$ is a probability on $X$ for $q \in \mathbf{Q}$, find a probability $\zeta$ on $\mathscr{H}$ (or, by extension, a probability $\zeta$ on $\mathscr{D}$ satisfying $\zeta(\mathbf{H})=1)$ such that $\Pi_{\mathrm{q}}(\mathbf{C})=\zeta(\mathbf{H}(\mathrm{q}, \mathbf{C})$ ) for $\mathbf{C}$ $\in \mathfrak{X}, \mathrm{q} \in \mathbf{Q}$. [Alternately, find a probability $v$ on $\mathcal{U}$ such that $\Pi_{\mathrm{q}}(\mathbf{C})=v(\mathbf{U}(\mathrm{q}, \mathbf{C}))$ for $\mathbf{C} \in \mathfrak{X}, \mathrm{q} \in \mathbf{Q}$ ].

2.3.2. The Revealed Dominating Distribution Problem. If $\Pi_{\mathrm{q}}{ }^{-}$and $\Pi_{\mathrm{q}}^{+}$are non-negative bounded set functions on $\mathfrak{X}$ for $\mathrm{q} \in \mathbf{Q}$, find a probability $\zeta$ on $\mathscr{H}$ such that $\Pi_{\mathrm{q}}-(\mathbf{C}) \leq \zeta(\mathbf{H}(\mathrm{q}, \mathbf{C})) \leq \Pi_{\mathrm{q}}^{+}(\mathbf{C})$ for $\mathbf{C} \in$ $\mathfrak{X}, \mathrm{q} \in \mathbf{Q}$. [Alternately, find a probability $v$ on $\mathcal{U}$ with $\Pi_{\mathrm{q}}(\mathbf{C}) \leq v(\mathbf{U}(\mathrm{q}, \mathbf{C})) \leq \Pi_{\mathrm{q}}^{+}(\mathbf{C})$ for $\mathbf{C} \in \mathfrak{X}, \mathrm{q} \in \mathbf{Q}$ ].

2.4. The Axiom of Revealed Stochastic Preference [ARSP]: For a class $H$ of hypothesized decision rules, or alternately, for a class $\mathbf{U}$ of hypothesized utility functions, and for each finite sequence of trials $\mathbf{t}=<\left(\mathrm{q}_{1}, \mathbf{C}_{1}\right), \ldots,\left(\mathrm{q}_{\mathrm{m}}, \mathbf{C}_{\mathrm{m}}\right)>$ with $\mathbf{C}_{\mathrm{i}} \in \mathfrak{X}$ and $\mathrm{q}_{\mathrm{i}} \in \mathbf{Q}$,

$$
\sum_{i=1}^{m} \Pi_{q_{i}}\left(\boldsymbol{C}_{i}\right) \leq \alpha_{\mathrm{H}}(\mathbf{t})=\max _{\mathrm{d} \in \mathbf{H}} \sum_{i=1}^{m} \quad \mathbf{1}\left(\mathbf{d}\left(\mathrm{q}_{\mathrm{i}}\right) \subseteq \mathbf{C}_{\mathrm{i}}\right)
$$

or alternately,

$$
\sum_{i=1}^{m} \Pi_{q_{i}}\left(C_{i}\right) \leq \alpha_{\mathrm{U}}(\mathbf{t})=\max _{\mathrm{u} \in \mathrm{U}} \sum_{i=1}^{m} \quad \mathbf{1}\left(\mathbf{d}\left(\mathrm{q}_{\mathrm{i}} ; \mathrm{u}\right) \subseteq \mathbf{C}_{\mathrm{i}}\right) .
$$

The expressions $\alpha_{\mathrm{H}}(\mathbf{t})$ and $\alpha_{\mathrm{U}}(\mathbf{t})$ are, respectively, the H-intersection number and $\mathrm{U}$-intersection number for the trial sequence $\mathbf{t}$. When $\mathrm{H}$ is U-rational, these numbers coincide. More generally, the axiom may be applied to hypothesized decision rules $\mathbf{H}$ that are not necessarily obtained from utility maximization. ARSP says that the sum of choice probabilities over a finite sequence of trials is no larger 
than the maximum number of successes that an admissible decision rule [alternately, an admissible utility function] can produce. A central result for the revealed distribution problem, due to McFadden and Richter (1971), is that under some regularity conditions, ARSP is necessary and sufficient for consistency of observed choice probabilities with a specified theory of choice behavior.

\section{Finite Families of Choice Situations}

3.1. Discussion. In psychometric and voting applications, as well as discrete choice applications in economics and marketing, it is natural to consider choice situations in which the space of possible alternatives is finite. The classical economic choice problem can also be interpreted as finite when the index set $\mathbf{Q}$ of budgets is finite, so that $\mathbf{X}$ can be partitioned into a finite family of subsets $\left\{\mathbf{X}_{1}, \ldots, \mathbf{X}_{\mathrm{m}}\right\}$ with the property for each $\mathrm{i}=1, \ldots, \mathrm{m}$ and $\mathrm{q} \in \mathbf{Q}$, either $\mathbf{X}_{\mathrm{i}} \subseteq \mathbf{B}(\mathrm{q})$ or $\mathbf{X}_{\mathrm{i}} \cap \mathbf{B}(\mathrm{q})=\varnothing$, and $\mathfrak{X}$ is the field generated by the partition. In this case, observations provide no information on choice behavior within partition sets, so that the partition sets can themselves be defined as the objects of choice.

Throughout this section, we will define the index set $\mathbf{Q}$ as the family of feasible "budget sets" in $\mathbf{X}$, and name singleton sets by their elements, so that $\Pi_{\mathbf{B}}(\mathrm{x})$ denotes a choice probability for $\mathbf{C}=\{\mathrm{x}\}$ when $\mathbf{B}$ is a feasible choice set in the family $\mathbf{Q}$, and by construction $\Pi_{\mathbf{B}}(\mathrm{x})=0$ for $\mathrm{x} \notin \mathbf{B}$. Note that if $\mathbf{X}$ contains $m$ elements, then there are $m$ ! possible total orders of these elements. We will represent these orders by the finite family $\mathbf{U}$ of utility functions from $\mathbf{X}$ onto the integers $\{1, \ldots, \mathrm{m}\}$; note that this definition excludes ties, so that utility-maximizing choice functions will be decisive.

The revealed stochastic preference problem was originally examined for the case of $\mathbf{X}$ finite by Marschak (1960), Block and Marschak (1960), and Luce and Suppes (1965), and it is for this case that the most complete characterizations of a solution have been given, by McFadden and Richter (1971, 1990), Falmagne (1978), and Barbara (1990). A closely related result with a different application was obtained by Freedman and Purves (1969). We will need several definitions.

\subsection{Definitions.}

3.2.1. A set $\mathbf{Q}$ of choice situations forms a net if for every feasible set of alternatives, every larger set contained in $\mathbf{X}$ is also feasible; i.e., if $\mathbf{B} \in \mathbf{Q}$ and $\mathbf{A} \subseteq \mathbf{X} \backslash \mathbf{B}$, then $\mathbf{B} \cup \mathbf{A} \in \mathbf{Q}$. A set of choice situations is exhaustive if it forms a net and it contains each singleton in $\mathbf{X}$. 
3.2.2. Suppose choice situations $\mathbf{Q}$ form a net. Let $\#(\mathbf{A})$ denote the number of elements in a subset $\mathbf{A}$ of $\mathbf{X}$. For $\mathrm{x} \in \mathbf{X} \backslash \mathbf{A} \in \mathbf{Q}$, the $\underline{\text { Block-Marschak polynomial }} \mathrm{K}_{\mathrm{x}, \mathbf{A}}$ is the function

$$
\mathrm{K}_{\mathrm{x}, \mathbf{A}}=\sum_{i=0}^{\#(\boldsymbol{A})}(-1)^{\#(\mathrm{~A})-\mathrm{i}} \sum_{\boldsymbol{C} \subseteq \boldsymbol{A} \& \#(\boldsymbol{C})=i} \Pi_{\mathrm{X} \backslash \mathbf{C}}(\mathrm{x}) .
$$

The Block-Marschak polynomials can also be defined recursively, with

$$
\begin{aligned}
& \mathrm{K}_{\mathrm{x}, \boldsymbol{\odot}}=\Pi_{\mathrm{X}}(\mathrm{x}), \\
& \mathrm{K}_{\mathrm{x}, \mathbf{A}}=\Pi_{\mathbf{X} \backslash \mathbf{A}}(\mathrm{x})-\sum_{\boldsymbol{C} \subset \boldsymbol{A}} \mathrm{K}_{\mathrm{x}, \mathbf{C}},
\end{aligned}
$$

for all $\mathbf{A} \subseteq \mathbf{X} \backslash \mathbf{B}$ and $\mathbf{B} \in \mathbf{Q}$; see Falmagne (1978, Theorem 2). An implication of this construction is $\Pi_{\mathbf{B}}(\mathrm{x})=\sum_{\boldsymbol{C} \subseteq \boldsymbol{X} \backslash \boldsymbol{B}} \mathrm{K}_{\mathrm{x}, \mathbf{C}}$ for $\mathbf{B} \in \mathbf{Q}$. When the choice probabilities are the result of utility maximization, Barbara and Pattanaik (1986) provide a useful interpretation of $\mathrm{K}_{\mathrm{x}, \mathbf{A}}$ as the probability of the event that $\mathrm{X}$ is ranked behind the elements of $\mathbf{A}$ and ahead of all the remaining elements in $\mathbf{X} \backslash \mathbf{A}$.

For a trial $(\mathbf{B}, \mathrm{x})$ with $\mathrm{x} \in \mathbf{B} \in \mathbf{Q}$, and for $\mathrm{u} \in \mathbf{U}$, define $\mathrm{a}_{\mathbf{B}, \mathrm{x}, \mathrm{u}}=\mathbf{1}\left(\mathrm{x}=\operatorname{argmax}_{\mathrm{x}^{\prime} \in \mathbf{B}} \mathrm{u}\left(\mathrm{x}^{\prime}\right)\right)$. Form a column vector $\pi$ composed of subvectors for each $\mathbf{B} \in \mathbf{Q}$, with each subvector composed of the choice probabilities $\Pi_{\mathbf{B}}(\mathrm{x})$ for $\mathrm{x} \in \mathbf{B}$. Form the matrix $\mathbf{A}$ with element $\mathrm{a}_{\mathbf{B}, \mathrm{x}, \mathrm{u}}$ in the row corresponding to the trial $(\mathbf{B}, \mathrm{x})$ and column $\mathrm{u}$ for $\mathrm{u} \in \mathbf{U}$. An element of $\mathbf{A}$ is one if the associated trial is a success for the specified utility function, and is zero otherwise. Then, integer-weighted column sums of $\mathbf{A}$ will be the number of successes attainable for a specified trial sequence (with repetitions given by the integer weights) for the various utility functions, and the maximum of these column sums will be the U-intersection number for the trial sequence.

3.3. Theorem. If $\mathbf{X}$ is finite, $\mathbf{U}$ is the class of utility functions that totally order $\mathbf{X}, \mathbf{Q}$ is a family of choice situations, with $\mathbf{B} \in \mathbf{Q}$ a non-empty subset of $\mathbf{X}$, and $\Pi_{\mathbf{B}}(\mathrm{x})$ is a choice probability for $\mathbf{x} \in \mathbf{B} \in \mathbf{Q}$ satisfying $\Pi_{\mathbf{B}}(\mathbf{B})=1$, then the following conditions are equivalent:

(a) There exists a probability $v$ on $\mathcal{U}$ that rationalizes the choice probability; i.e., 


$$
\Pi_{\mathbf{B}}(\mathrm{x})=\sum_{u \in \boldsymbol{U}} \mathrm{a}_{\mathbf{B}, \mathrm{x}, \mathrm{u}} v_{\mathrm{u}} \text { for } \mathrm{x} \in \mathbf{B} \in \mathbf{Q} .
$$

(b) The system of linear inequalities $\pi \leq \mathbf{A} v, v \geq 0, \mathbf{1}^{\prime} v \leq 1$ has a solution.

(c) The linear program $\min _{v, s} \mathbf{1}^{\prime}$ s subject to $v \geq 0, \mathrm{~s} \geq 0, \mathbf{A} v+\mathrm{s} \geq \pi, \mathbf{1}^{\prime} v \leq 1$ has an optimal solution with $\mathrm{s}=0$.

(d) The linear program $\max _{\mathrm{r}, \mathrm{t}}\left(\mathrm{r}^{\prime} \pi-\mathrm{t}\right)$ subject to $0 \leq \mathrm{r} \leq 1, \mathrm{t} \geq 0$, and $\mathrm{r}^{\prime} \mathbf{A} \leq \mathrm{t} \mathbf{1}^{\prime}$ has no positive solution.

(e) The choice probabilities $\Pi_{\mathbf{B}}(\mathrm{x}), \mathrm{x} \in \mathbf{B} \in \mathbf{Q}$, satisfy ARSP [cf 2.4].

If the set $\mathbf{Q}$ of feasible choice situations forms a net, then (a)-(e) are equivalent to

(f) The Block-Marschak polynomials $\mathrm{K}_{\mathrm{x}, \mathbf{X I B}}$ for $\mathrm{x} \in \mathbf{B} \in \mathbf{Q}$, are non-negative.

Proof: If a probability $v$ satisfies (a), then it satisfies (b) with $\pi=\mathbf{A} v$. Conversely, if $\pi$ satisfies (b), then $\pi=\mathbf{A} v$ since $\pi$ satisfies $\Pi_{\mathbf{B}}(\mathbf{B})=1$, so that (a) is satisfied. But $v$ solves (b) if and only if $v$ and $\mathrm{s}=0$ solve (c). The linear program (d) is dual to the linear program (c), so that (c) has an optimal solution with $\mathrm{s}=0$ if and only if (d) has no positive solution; see Karlin (1959, V.4.1). An optimal solution to (d) satisfies $t=\max _{u} r^{\prime} A_{u}$, where $A_{u}$ is a column of $\mathbf{A}$. Thus, (d) has a positive optimal solution if and only if for some $r$ satisfying $0 \leq r \leq 1$, one has $r^{\prime} \pi>t=\max _{u} r^{\prime} A_{u}$. But if this is true, then one can achieve the strict inequality with a vector $r$ whose components are all rational numbers. Clear a common denominator so that $r$ is a vector of non-negative integers. Then, $\max _{u} r^{\prime} A_{u}$ is the intersection number of the sequence of trials with the components of $r$ giving the number of repetitions for each trial, so that (d) has a positive solution if and only if ARSP in (e) is violated. This establishes that (a)-(e) are equivalent.

Consider condition (f), and suppose $\mathbf{Q}$ forms a net so that the Block-Marschak polynomials $\mathrm{K}_{\mathrm{x}, \mathbf{A}}$ are defined for $\mathrm{x} \in \mathbf{X} \backslash \mathbf{A} \in \mathbf{Q}$. Let $\mathbf{r}=\left\langle\mathrm{r}_{1}, \ldots, \mathrm{r}_{\mathrm{k}}>\right.$ denote an ordered sequence of the elements of a set $\mathbf{A}=$ $\left\{r_{1}, \ldots, r_{k}\right\} \subseteq \mathbf{X}$, where $\mathrm{k}=0, \ldots, \#(\mathbf{X})$, and $\mathbf{R}_{\mathbf{A}}$ denote the family of all ordered sequences $\mathbf{r}$ of the elements of $\mathbf{A}$. Let $\mathbf{B} \backslash \mathbf{r}$ denote the set of elements of $\mathbf{B}$ that are not contained in the sequence $\mathbf{r}$. For $\mathbf{r} \subseteq \mathbf{B}$, define

$$
\mathbf{S}_{\mathrm{r}, \mathbf{B}}=\left\{\mathrm{u} \in \mathbf{U} \mid \mathrm{u}\left(\mathrm{r}_{1}\right)>\ldots>\mathrm{u}\left(\mathrm{r}_{\mathrm{k}}\right)>\mathrm{u}(\mathrm{x}) \text { for } \mathrm{x} \in \mathbf{B} \backslash \mathbf{r}\right\}
$$


Then, $\mathbf{S}_{\mathbf{r}, \mathbf{B}}$ contains the utility functions for which the elements in $\mathbf{r}$ are ranked in descending order and are better than any remaining elements in B. If (a) holds, it is immediate from the construction of $\mathbf{S}_{\mathbf{r}, \mathbf{B}}$ that for $\mathbf{x} \in \mathbf{B} \in \mathbf{Q}, \Pi_{\mathbf{B}}(\mathrm{x})=v\left(\mathbf{S}_{<\mathbf{x}>\mathbf{B}}\right)$. The sets $\mathbf{S}_{\mathbf{r}, \mathbf{B}}$ for $\mathbf{B} \in \mathbf{Q}$ have the property that $\mathbf{S}_{<\mathbf{r}, \mathbf{x}>\mathbf{B}}$ for $\mathrm{x} \in \mathbf{B} \backslash \mathbf{r}$ is a partition of $\mathbf{S}_{\mathbf{r}, \mathbf{B}}$ (Falmagne, 1978, Lemma 1) and for $\mathbf{x} \in \mathbf{B} \in \mathbf{Q}$ and $\mathbf{A}=\mathbf{X} \backslash \mathbf{B}, \mathbf{S}_{<\mathbf{x}>, \mathbf{B}}=\cup_{\boldsymbol{r} \in \boldsymbol{K}} \mathbf{S}_{<\mathbf{r}, \mathbf{x}>\mathbf{X}, \mathbf{X}}$,

with the sets in this union disjoint (Falmagne, 1978, Lemma 2). Note that $\bigcup_{x \in I} \mathbf{S}_{<\mathbf{x}>\mathbf{x}}=\mathbf{U}$. The family

of sets $\mathscr{J}_{0}=\left\{\mathbf{S}_{<\mathbf{r}, \mathbf{x}>\mathbf{x}} \mid \mathbf{x} \in \mathbf{B} \in \mathbf{Q}\right.$ and $\mathbf{r} \in \mathbf{R}_{\mathbf{C}}$ for $\left.\mathbf{C} \subseteq \mathbf{X} \backslash \mathbf{B}\right\}$ then form a Boolean semi-algebra (Neveu (1965, 1.6.1). Consider the sets $\mathbf{M}_{\mathrm{x}, \mathbf{A}}=\left\{\mathrm{u} \in \mathbf{U} \mid \mathrm{u}\left(\mathrm{x}^{\prime}\right)>\mathrm{u}(\mathrm{x})>\mathrm{u}\left(\mathrm{x}^{\prime \prime}\right)\right.$ for $\mathrm{x}^{\prime} \in \mathbf{A}$ and $\left.\mathrm{x} \neq \mathrm{x}^{\prime \prime} \in \mathbf{X} \backslash \mathbf{A}\right\}=\bigcup_{r \leq \mathbf{l}} \mathbf{S}_{<\mathrm{r}, \mathrm{x}>\mathrm{x}, \mathrm{x}}$ and note that the sets in the last union are disjoint. Barbara and Pattanaik (1986, Theorem 2.1) utilize the recursive definition of $\mathrm{K}_{\mathrm{x}, \mathbf{A}}$ to prove by induction for $\mathrm{x} \in \mathbf{X} \backslash \mathbf{A} \in \mathbf{Q}$ that when (a) holds,

$$
\mathrm{K}_{\mathrm{x}, \mathbf{A}}=v\left(\mathbf{M}_{\mathrm{x}, \mathbf{A}}\right)=\sum_{\boldsymbol{r} \subseteq \boldsymbol{R}_{A}} v\left(\mathbf{S}_{<\mathbf{r}, \mathbf{x}>\mathbf{x}}\right) \geq 0
$$

Then, (a) implies (f).

Suppose that the Block-Marschak polynomials are non-negative for a class of feasible choice sets $\mathbf{Q}$ that forms a net, so that (f) holds. Following Falmagne (1978, Theorem 4), construct a set-valued function $v$ on $\mathscr{J}_{0}$ in the following steps:

(1) For $\mathrm{x} \in \mathbf{X}, v\left(\mathbf{S}_{<\mathbf{x}>, \mathbf{x}}\right)=\mathrm{K}_{\mathrm{x}, \boldsymbol{\ominus}} \equiv \Pi_{\mathbf{X}}(\mathrm{x})$.

(2) For $\mathrm{x}, \mathrm{y} \in \mathbf{X}, \mathrm{x} \neq \mathrm{y}, \boldsymbol{v}\left(\mathbf{S}_{<\mathrm{y}, \mathrm{x}>, \mathbf{x}}\right)=\mathrm{K}_{\mathrm{x},\{\mathrm{y}\}} \equiv \Pi_{\mathbf{X} \backslash\{\mathrm{y}\}}(\mathrm{x})-\Pi_{\mathbf{X}}(\mathrm{x})$.

(3) Suppose $v$ has been defined for $\mathbf{S}_{\mathbf{r}, \mathbf{X}}$ with $\mathbf{r} \in \mathbf{R}_{\mathbf{A}}$ for all $\mathbf{A}$ such that $\mathbf{X} \backslash \mathbf{A} \in \mathbf{Q}$ and $\#(\mathbf{A})<\mathrm{k}$. Suppose A meets this condition with \#(A)=k-1, and suppose $\mathrm{x} \in \mathbf{X} \backslash \mathbf{A}$ satisfies $(\mathbf{X} \backslash \mathbf{A}) \cup \mathbf{X} \in \mathbf{Q}$. Define $\Delta=\sum_{\boldsymbol{r} \in \boldsymbol{R}_{A}} v\left(\mathbf{S}_{\mathbf{r}, \mathbf{X}}\right)$. Then, define $v\left(\mathbf{S}_{<\mathbf{r}, \mathbf{x}>\mathbf{X}}\right)$ by the recursion $v\left(\mathbf{S}_{<\mathbf{r}, \mathbf{x}>\mathbf{X}}\right)=\mathrm{K}_{\mathbf{x}, \mathbf{A}} \cdot v\left(\mathbf{S}_{\mathbf{r}, \mathbf{X}}\right) / \Delta$ if $\Delta>0$, and otherwise $v\left(\mathbf{S}_{<\mathbf{r}, \mathbf{x}>, \mathbf{X}}\right)=0$.

It is immediate from this construction and the fact that $\mathbf{S}_{<\mathbf{r}, \mathbf{x}>\mathbf{X}}$ is a partition of $\mathbf{S}_{\mathbf{r}, \mathbf{X}}$ for $\mathbf{x} \in \mathbf{X} \backslash \mathbf{r}$ that $v$ is non-negative and additive on $\mathscr{J}_{0}$, with $v(\mathbf{U})=1$; see Falmagne (1978, Lemma 4). Then $v$ has a unique extension to a probability on the Boolean algebra $\mathscr{J}$ generated by $\mathfrak{J}_{0}$ (Neveu, 1964, 1.6.1). Further, 
defining $v(\mathbf{A})=\sup \{v(\mathbf{B}) \mid \mathbf{B} \in \mathfrak{I} \& \mathbf{B} \subseteq \mathbf{A}\}$ for $\mathbf{A} \subseteq \mathbf{U}$ extends $v$ to a probability on the Boolean algebra of all subsets of $\mathbf{U}$; see Neveu (1965, I.6.2). The final step of the proof is to show that the constructed probability $v$ satisfies (a). Since $\Pi_{\mathrm{B}}(\mathrm{x})=\sum_{C \subseteq X \backslash B} \quad \mathrm{~K}_{\mathrm{x}, \mathrm{C}}$ for $\mathbf{B} \in \mathbf{Q}$, it is sufficient to show that $\mathrm{K}_{\mathrm{x}, \mathbf{A}}$ $=v\left(\mathbf{M}_{\mathrm{x}, \mathbf{A}}\right)=\sum_{r \subseteq A} v\left(\mathbf{S}_{<\mathrm{r}, \mathrm{x}>\mathrm{X}}\right)$ for $\mathbf{X} \backslash \mathbf{A} \in \mathbf{Q}$. But the construction $v\left(\mathbf{S}_{<\mathrm{r}, \mathrm{x}, \mathbf{X}}\right)=\mathrm{K}_{\mathrm{x}, \mathbf{A}} \cdot v\left(\mathbf{S}_{\mathrm{r}, \mathbf{X}}\right) / \Delta$ implies $\sum_{\boldsymbol{r} \in \boldsymbol{R}_{\boldsymbol{A}}} v\left(\mathbf{S}_{<\mathrm{r}, \mathbf{x}, \mathbf{X}}\right)=\mathrm{K}_{\mathrm{x}, \mathbf{A}}$. This completes the proof.

3.4. Remarks. The equivalence of (a)-(e) was established by McFadden and Richter $(1971,1990)$. The equivalence of (a) and (f) when the family of feasible choice sets is exhaustive was established by Falmagne (1978), with useful interpretation and refinements given by Barbara and Pattianiak (1986). Theorem 3.3 generalizes the Falmagne result slightly by noting that it is not necessary that the feasible choice sets be exhaustive, provided that they form a net so that the Block-Marschak polynomials are defined.

The linear programs (c) and (d) provide finite algorithms that can, in principle, determine if observed choice probabilities can be rationalized. These are, further, completely general, requiring no particular structure for the set of feasible choice situations. The construction for condition (f) is also a finite algorithm, with the advantage that each step in the recursive construction of the measure $v$ defines a probability on a Boolean semi-algebra of subsets of U. In some applications, such as construction of bounds, this intermediate information may be directly useful. The primary limitation of the Block-Marschak polynomial condition is that it requires that the feasible choice sets form a net. This excludes some natural applications, such as those where only paired comparisons are observed, or those defined by economic budget sets for a finite number of price vectors.

Part of the literature on stochastic choice has concentrated on situations where decision-makers are faced with binary choice situations; see Luce (1959), McLennon (1991), Fishburn (1999). Falmagne's condition on the Block-Marschak polynomials is not applicable to this case, and while ARSP is applicable, it does not fully exploit the geometry of the polytope containing the vectors of rationalizable 
choice probabilities. Fishburn (1992) surveys the results on this problem, including the mathematical literature on the polytopes generated by the decisive preference preorders.

\section{Extension of Set Functions}

4.1. The Dominance Problem. The results of this paper for the non-finite case hinge on the following mathematical problem: If $\mathrm{P}$ is a non-negative bounded set function on a family $\varsigma$ of subsets of a non-empty set $\mathbf{H}$, find a probability $\eta$ on the Boolean algebra $ฮ$ generated by $\S$ such that $\eta(\mathbf{S}) \geq \mathrm{P}(\mathbf{S})$ for $\mathbf{S} \in 5$. The following axiom is the key to the existence of a solution..

4.2. The Dominance Axiom. For each finite sequence $\mathbf{t}=\left\langle\mathbf{S}_{1}, \ldots, \mathbf{S}_{\mathrm{m}}>\right.$ in $\delta$, with repetitions allowed,

$$
\sum_{i=}^{m} \mathrm{P}\left(\mathrm{S}_{\mathrm{i}}\right) \leq \alpha_{\mathbf{H}}(\mathbf{t})=\max _{\mathrm{d} \in \mathbf{H}} \sum_{i=}^{m} \mathbf{1}\left(\mathrm{d} \in \mathbf{S}_{\mathrm{i}}\right)
$$

4.3. Finitely-Additive Extension Theorem.. $P$ is a non-negative bounded set function satisfying the dominance axiom on a family $\varsigma$ of subsets of a non-empty set $\mathbf{H}$ if and only if there exists a finitely additive probability $\eta$ on a Boolean algebra $\mathscr{J}$ of subsets of $\mathbf{H}$ containing $\varsigma$ such that $\eta(\mathbf{S}) \geq \mathrm{P}(\mathbf{S})$ for $\mathbf{S}$ $\in$ ฮ. If, further, $\varsigma$ is closed under complementation and contains $\mathbf{H}$, and $\mathrm{P}$ satisfies $\mathrm{P}(\mathbf{S})+\mathrm{P}\left(\mathbf{S}^{\mathrm{c}}\right)=1$ for $\mathbf{S} \in \delta$, then $\eta(\mathbf{S})=\mathrm{P}(\mathbf{S})$ for $\mathbf{S} \in \mathcal{\delta}$.

Proof: Necessity of the dominance axiom: . Let $\mathbf{S}_{1}, \ldots, \mathbf{S}_{\mathrm{n}}$ be a sequence of sets in $\delta$, and $\mathbf{T}_{1}, \ldots, \mathbf{T}_{\mathrm{m}}$ the partition of $\mathbf{H}$ that they induce. Then $\mathbf{T}_{j} \in \mathcal{J}$. Let $k_{j}$ equal the number of sets $\mathbf{S}_{\mathrm{i}}$ containing $\mathbf{T}_{\mathrm{j}}$. Then

$$
\sum_{i=1}^{n} \mathrm{P}\left(\mathbf{S}_{\mathrm{i}}\right) \leq \sum_{i=1}^{n} \eta\left(\mathbf{S}_{\mathrm{i}}\right)=\sum_{j=1}^{m} \mathrm{k}_{\mathrm{j}} \cdot \eta\left(\mathbf{T}_{\mathrm{j}}\right) \leq \max _{\mathrm{j} \leq \mathrm{m}} \mathrm{k}_{\mathrm{j}} \equiv \alpha_{\mathrm{D}}\left(<\mathbf{S}_{1}, \ldots, \mathbf{S}_{\mathrm{n}}>\right) .
$$

Sufficiency of the dominance axiom: Suppose $\mathbf{P}$ satisfies the dominance axiom. Let $\mathbf{Y}$ denote the linear space spanned by the indicator functions $\mathbf{1}_{\mathbf{S}}$ of the sets $\mathbf{S} \in \mathfrak{J}$, and $\mathbf{Z}$ denote its linear subspace spanned by the indicator functions $\mathbf{1}_{\mathbf{S}}$ of the sets $\mathbf{S} \in \delta$. Define on $\mathbf{Y}$ the norm $\|\mathrm{f}\|=\sup _{\mathrm{d} \in \mathbf{H}}|\mathrm{f}(\mathrm{d})|$. Define a convex cone in $\mathbf{Z}$, 


$$
\mathbf{W}=\left\{\mathrm{f} \in \mathbf{Z} \mid \mathrm{f}=\sum_{i=1}^{m} k_{i} \mathbf{1}_{i} \text { for } \mathrm{m}>0, \text { non-negative scalars } \mathrm{k}_{\mathrm{i}} \text {, and } \mathbf{S}_{\mathrm{i}} \in \varsigma\right\},
$$

and on $\mathbf{W}$ define the functional

$$
\mathrm{p}(\mathrm{f})=\sup \left\{\sum_{i=}^{m} \mathrm{k}_{\mathrm{i}} \mathrm{P}\left(\mathbf{S}_{\mathrm{i}}\right) \mid \mathrm{f}=\sum_{i=1}^{m} k_{i} \mathbf{1}_{i} \text { for } \mathrm{m}>0, \text { non-negative scalars } \mathrm{k}_{\mathrm{i}} \text {, and } \mathbf{S}_{\mathrm{i}} \in \delta\right\}
$$

On the space $\mathbb{R} \times \mathbf{Z}$ with the norm $|\mathrm{r}|+\|\mathrm{f}\|$ for $(\mathrm{r}, \mathrm{f}) \in \mathbb{R} \times \mathbf{Z}$, define the sets $\mathbf{A}_{1}=\{(\mathrm{r}, \mathrm{f}) \in \mathbb{R} \times \mathbf{Z} \mid \mathrm{r} \geq\|\mathrm{f}\|\}$ and $\mathbf{A}_{2}$ $=\{(\mathrm{r}, \mathrm{f}) \in \mathbb{R} \times \mathbf{W} \mid \mathbf{r}<\mathrm{p}(\mathrm{f})\}$. Then $\mathbf{A}_{1}$ and $\mathbf{A}_{2}$ are convex cones, and $\mathbf{A}_{1}$ has a non-empty interior in the norm topology of $\mathbf{Z}$. Suppose $\mathbf{A}_{1}$ and $\mathbf{A}_{2}$ have a common point $\left(\mathrm{r}^{0}, \mathrm{f}^{0}\right)$. Then, $\left\|\mathrm{f}^{0}\right\| \leq \mathrm{r}^{0}<\mathrm{p}\left(\mathrm{f}^{0}\right)-\varepsilon$ for some positive $\varepsilon$. From the definition of $\mathrm{p}(\mathrm{f})$, there exists a representation $\mathrm{f}^{0}=\sum_{i=1}^{m} k_{i} \mathbf{1}_{S}$ such that $\mathrm{p}\left(\mathrm{f}^{0}\right) \leq$

$$
\sum_{i=1}^{m} k_{i} \mathrm{P}\left(\mathbf{S}_{\mathrm{i}}\right)+\varepsilon / 2 \text {. Then } \sup _{\mathrm{d} \in \mathbf{H}} \sum_{i=1}^{m} k_{i} \mathbf{1}_{S_{i}}\left(d<\sum_{i=}^{m} k_{i} \mathrm{P}\left(\mathbf{S}_{\mathrm{i}}\right)-\boldsymbol{\varepsilon} / 2\right. \text {. Since this inequality is }
$$

continuous in the $k_{i}$, these numbers can be chosen to be rational, and a common denominator cleared so that the inequality $\sup _{\mathrm{d} \in \mathbf{H}} \sum_{i=1}^{m} k_{i} \mathbf{1}_{S_{i}}(d)<\sum_{i=:}^{m} k_{i} \mathrm{P}\left(\mathbf{S}_{\mathrm{i}}\right)$ holds for some $k_{i}$ integral. Considering a sequence of sets $\mathbf{S}_{\mathrm{i}}$ with repetitions $k_{i}$ for $\mathrm{i}=1, \ldots, \mathrm{m}$ gives a violation of the dominance axiom. Hence, $\mathbf{A}_{1}$ and $\mathbf{A}_{2}$ are disjoint. A separating hyperplane theorem (Dunford and Schwartz (1964, V.2.8) implies the existence of a non-zero continuous linear functional $(\lambda, \zeta)$ on $\mathbb{R} \times \mathbf{Z}$ such that $\lambda \mathrm{r}-\zeta(\mathrm{f}) \geq 0$ for $(\mathrm{r}, \mathrm{f}) \in$ $\mathbf{A}_{1}$ and $\lambda \mathrm{r}-\zeta(\mathrm{f}) \leq 0$ for $(\mathrm{r}, \mathrm{f}) \in \mathbf{A}_{2}$. If $\lambda \leq 0$, the first inequality holds at $(1,0) \in \mathbf{A}_{1}$ only if $\lambda=0$. However, $\lambda=0$ requires $\zeta(\mathrm{f}) \leq 0$ for all $\mathrm{f} \in \mathbf{Z}$, implying $\zeta(\mathrm{f}) \equiv 0$, a contradiction of $(\lambda, \zeta)$ non-zero. Hence, $\lambda>0$, and we can normalize it to one. Then, the first inequality implies $\|\mathrm{f}\| \geq \zeta(\mathrm{f})$ on $\mathbf{Z}$, while the second inequality implies $\zeta\left(\mathbf{1}_{\mathbf{S}}\right) \geq \mathrm{P}(\mathbf{S})$ for $\mathbf{S} \in \mathcal{~}$. The Hahn-Banach theorem implies $\zeta$ can be extended to a linear functional on $\mathbf{Y}$ satisfying $\zeta(f) \leq\|f\|$. Then $\eta(\mathbf{S})=\zeta\left(\mathbf{1}_{\mathbf{s}}\right)$ is a finitely additive probability satisfying the dominance condition $\eta(\mathbf{S}) \geq \mathrm{P}(\mathbf{S})$ for $\mathbf{S} \in \mathcal{\varsigma}$.

If $\mathbf{S}$ is closed under complementation and $\mathrm{P}(\mathbf{S})+\mathrm{P}\left(\mathbf{S}^{\mathrm{c}}\right)=1$ for $\mathbf{S} \in \mathcal{\varsigma}$, then the inequality $1=\mathrm{P}(\mathbf{S})+$ $\mathrm{P}\left(\mathbf{S}^{\mathrm{c}}\right) \leq \eta(\mathbf{S})+\eta\left(\mathbf{S}^{\mathrm{c}}\right)=1$ implies $\eta(\mathbf{S})=\mathrm{P}(\mathbf{S})$ for $\mathbf{S} \in$ 厄. 
4.4. Compact families. A family $\mathscr{K}$ of subsets of a set $\mathbf{H}$ is compact if every sequence of members with the finite intersection property has a non-empty intersection. The family formed from $\mathscr{K}$ by the operations of finite union and countable intersection is again compact (Neveu, 1965, 1.6.1).

4.5. Tightness. Suppose a non-negative bounded set function $P$ is defined on a family $\S$ of subsets of a set $\mathbf{H}$. Suppose that $\varsigma$ is closed under complementation and contains $\mathbf{H}$, and that $\mathrm{P}(\mathbf{S})+\mathrm{P}\left(\mathbf{S}^{\mathrm{c}}\right)=1$ for $\mathbf{S} \in \mathcal{\delta}$. The function $P$ is tight if there is a compact family of subsets $\mathscr{K}$ of $\mathbf{H}$ such that for each $\epsilon>$ 0 and $\mathbf{S} \in \mathcal{S}$ there exist $\mathbf{S}^{\prime} \in \mathcal{\delta}$ and $\mathbf{K} \in \mathscr{K}$ such that $\mathbf{S}^{\prime} \subseteq \mathbf{K} \subseteq \mathbf{S}$ and $\mathrm{P}(\mathbf{S})-\mathrm{P}\left(\mathbf{S}^{\prime}\right)<\epsilon$.

The definition does not require that $\mathrm{P}(\mathbf{K})$ be defined for $\mathbf{K} \in \mathcal{K}$, but simplifies (to the requirement that $\mathrm{P}(\mathbf{S})$ - $\mathrm{P}(\mathbf{K})<\epsilon$ for some $\mathbf{K} \subseteq \mathbf{S}$ and $\mathbf{K} \in \mathscr{K}$ ) when $\mathscr{K} \subseteq \mathcal{S}$. If $\mathbf{S}$ is (almost surely) finite, it is itself a compact class. ${ }^{4}$ More generally, suppose $\mathbf{H}$ can be partitioned into "atoms" $\left\{\mathbf{H}_{1}, \ldots, \mathbf{H}_{\mathrm{N}}\right\}$ plus a non-atomic set $\mathbf{H}_{0}, \varsigma_{0}$ is a family of subsets of $\mathbf{H}_{0}$, and $\varsigma$ is a family whose members can be written as finite unions of sets in $\varsigma_{0}$ and the atoms $\mathbf{H}_{1}, \ldots, \mathbf{H}_{\mathrm{N}}$, or complements of such sets. If $\mathscr{K}_{0}$ is a compact class of subsets of $\mathbf{H}_{0}$ that is closed under countable intersection, then $\mathscr{K}$ formed by finite unions of $\mathbf{H}_{1}, \ldots, \mathbf{H}_{\mathrm{N}}$, and sets $\mathbf{K} \in$ $\mathscr{K}_{0}$ is again a compact class. Thus, $\mathrm{P}$ can satisfy our definition of tightness even if it has a finite number of atoms. When $\varsigma$ is a Boolean algebra and $\mathscr{K}$ is contained in $\varsigma$, our definition of tightness coincides with that of Neveu (1965, I.6.3). The following result relates tightness and countable additivity.

4.6. Lemma. If $\mathrm{P}$ is a non-negative, finitely additive set function defined on the Boolean algebra $\mathfrak{I}_{0}$ generated by a family $\S$ of subsets of a non-empty set $\mathbf{H}$, and if $\mathrm{P}$ is tight on $\S$, then $\mathrm{P}$ is countably additive on $\mathscr{I}_{0}$, and has a unique countably additive extension to the Boolean $\sigma$-algebra $\mathscr{J}$ generated by $\mathscr{J}_{0}$. Conversely, if $\mathrm{P}$ is countably additive on a Boolean $\sigma$-algebra $\mathfrak{I}$, then each of the following conditions is sufficient for it to be tight:

(a) $\mathbf{H}$ is a Polish space (i.e., a complete separable metric space) and $\mathscr{J}$ is its Borel $\sigma$-field.

(b) $\mathbf{H}$ is a compact Hausdorff space with a countable base, and $\mathscr{J}$ is its Borel $\sigma$-field.

(c) $\mathbf{H}$ is a countable space, and $\mathfrak{I}$ is the field of all subsets of $\mathbf{H}$.

\footnotetext{
${ }^{4}$ The class $\Subset$ is almost surely finite if it is countable, and $\mathrm{P}(\mathbf{S})=0$ for all except a finite number of sets $\mathbf{S}$ in $\nwarrow$.
} 
Proof: Suppose $\mathrm{P}$ is finitely additive on the Boolean algebra $\mathfrak{J}_{0}$ generated by a family of sets $\Subset$, and $\mathrm{P}$ is tight on $\widetilde{ }$. We show that $\mathrm{P}$ is tight on $\mathscr{J}_{0}$, and consequently $\sigma$-additive. First let $\mathbf{T}_{\mathrm{n}}=\bigcap_{i=1}^{n} \mathbf{S}_{\mathrm{i}}$ be a finite intersection of sets $\mathbf{S}_{\mathrm{i}} \in \mathcal{\delta}$. The tightness assumption on $\mathrm{P}$ implies there exists a compact class $\mathscr{K}$ of subsets of $\mathbf{H}$, which we take without loss of generality to be closed under finite union and countable intersection, such that given $\epsilon>0$ there exist $\mathbf{S}_{\mathrm{i}}{ }^{\prime} \subseteq \mathbf{C}_{\mathrm{i}} \subseteq \mathbf{S}_{\mathrm{i}}$ with $\mathbf{C}_{\mathrm{i}} \in \mathcal{K}$ and $\mathrm{P}\left(\mathbf{S}_{\mathrm{i}}\right)-\mathrm{P}\left(\mathbf{S}_{\mathrm{i}}{ }^{\prime}\right)<\epsilon \cdot 2^{-\mathrm{i}}$. The set $\bigcap_{i=1}^{n} \mathbf{C}_{\mathrm{i}}$ is in $\mathcal{K}$. The set inclusion $\left(\bigcap_{i=1}^{n} \mathbf{S}_{\mathrm{i}}\right) \backslash\left(\bigcap_{i=1}^{n} \mathbf{S}_{\mathrm{i}}{ }^{\prime}\right) \subseteq \bigcup_{i=1}^{n}\left(\mathbf{S}_{\mathrm{i}} \backslash \mathbf{S}_{\mathrm{i}}{ }^{\prime}\right)$ and the additivity and subadditivity of $\mathrm{P}$ imply

$$
\begin{aligned}
\mathrm{P}\left(\bigcap_{i=1}^{n} \mathbf{S}_{\mathrm{i}}\right)-\mathrm{P}\left(\bigcap_{i=1}^{n} \mathbf{S}_{\mathrm{i}}{ }^{\prime}\right)=\mathrm{P}\left(\left(\bigcap_{i=1}^{n} \mathbf{S}_{\mathrm{i}}\right) \backslash\left(\bigcap_{i=1}^{n} \mathbf{S}_{\mathrm{i}}{ }^{\prime}\right)\right) \\
\leq \sum_{i=1}^{n} \mathrm{P}\left(\mathbf{S}_{\mathrm{i}} \backslash \mathbf{S}_{\mathrm{i}}{ }^{\prime}\right)=\sum_{i=1}^{n}\left[\mathrm{P}\left(\mathbf{S}_{\mathrm{i}}\right)-\mathrm{P}\left(\mathbf{S}_{\mathrm{i}}{ }^{\prime}\right)\right] \leq \epsilon .
\end{aligned}
$$

Then $\mathrm{P}$ satisfies the definition for tightness on the family $\varsigma_{1}$ of all sets formed from $\varsigma$ by the operation of countable intersection. Next, consider the family $\varsigma_{2}$ of all sets formed from $\varsigma_{1}$ by the operation of finite union $\mathbf{V}=\bigcup_{j=1}^{N} \mathbf{T}_{\mathrm{j}}$ of pairwise disjoint sets $\mathbf{T}_{\mathrm{j}} \in \varsigma_{1}$. From the previous construction, there exist $\mathbf{C}_{\mathrm{j}} \in \mathcal{K}$ and $\mathbf{T}_{\mathrm{j}}{ }^{\prime} \in \varsigma_{1}$ satisfying $\mathbf{T}_{\mathrm{j}}{ }^{\prime} \subseteq \mathbf{C}_{\mathrm{j}} \subseteq \mathbf{T}_{\mathrm{j}}$ and $\mathrm{P}\left(\mathbf{T}_{\mathrm{j}}\right)-\mathrm{P}\left(\mathbf{T}_{\mathrm{j}}{ }^{\prime}\right) \leq \epsilon / \mathrm{N}$, implying $\bigcup_{j=1}^{N} \mathbf{K}_{\mathrm{j}} \in \mathcal{K}, \mathbf{V}^{\prime}=$ $\bigcup_{j=1}^{N} \mathbf{T}_{\mathrm{j}}{ }^{\prime} \in \varsigma_{2}$, and P(V) $-\mathrm{P}\left(\mathbf{V}^{\prime}\right) \leq \epsilon$. But $\varsigma_{2}=\mathfrak{J}_{0}($ Neveu, 1965 I.2.2), so that we have established that $\mathrm{P}$ is tight on $\mathfrak{J}_{0}$.

Suppose sets $\mathbf{V}_{\mathrm{n}}, \mathbf{V}_{\mathrm{n}}{ }^{\prime} \in \mathscr{J}_{0}$ and $\mathbf{C}_{\mathrm{n}} \in \mathscr{K}$ satisfy $\mathbf{V}_{\mathrm{n}}{ }^{\prime} \subseteq \mathbf{C}_{\mathrm{n}} \subseteq \mathbf{V}_{\mathrm{n}}, \mathrm{P}\left(\mathbf{V}_{\mathrm{n}}\right)-\mathrm{P}\left(\mathbf{V}_{\mathrm{n}}{ }^{\prime}\right) \leq \epsilon$, and $\mathbf{V}_{\mathrm{n}} \vee \varnothing$. Then, $\mathbf{C}_{\mathrm{n}}$ $\triangleright \varnothing$, and compactness implies there exists $\mathrm{N}$ such that $\mathbf{V}_{\mathrm{N}}{ }^{\prime} \subseteq \mathbf{C}_{\mathrm{N}}=\varnothing$. Then $\mathrm{P}\left(\mathbf{V}_{\mathrm{N}}{ }^{\prime}\right)=0$, implying $\mathrm{P}\left(\mathbf{V}_{\mathrm{N}}\right)$ $\leq \epsilon$, and $\mathrm{P}$ is continuous at $\varnothing$, and hence countably additive. The Hahn extension theorem (Dunford, 1964, III.5.8) establishes that P has a unique countably additive extension to the Boolean $\sigma$-algebra $\mathscr{J}$ generated by $\mathscr{J}_{0}$.

Consider the sufficient conditions for tightness. Condition (a) is given by Neveu (1965), II.7.3. Condition (b) reduces to condition (a) by the Urysohm metrization theorem. Condition (c) reduces to condition (a) by assigning $\mathbf{H}$ the metric $\rho(x, y)=\mathbf{1}(x \neq y)$. 
4.7. Countably Additive Extension Theorem. Suppose $\varsigma$ is a family of subsets of a non-empty set $\mathbf{H}$ that contains $\mathbf{H}$ and is closed under complementation, and $\mathrm{P}$ is a non-negative bounded set function

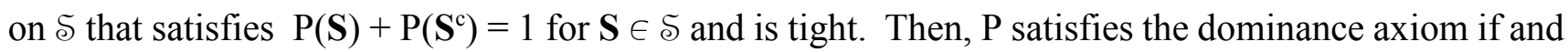
only if there exists a countably additive probability $\eta$ on the Boolean $\sigma$-algebra $\mathfrak{J}$ of subsets of $\mathbf{H}$ generated by $\varsigma$ such that $\eta(\mathbf{S})=\mathrm{P}(\mathbf{S})$ for $\mathbf{S} \in \delta$.

Proof: The proof of Theorem 4.3 establishes the existence of $\eta$ finitely additive on $\mathscr{J}$ and satisfying $\eta(\mathbf{S})$ $=\mathrm{P}(\mathbf{S})$ for $\mathbf{S} \in \delta$ if and only if the dominance axiom holds. This also establishes the necessity of the dominance axiom when $\eta$ is countably additive. For the sufficiency of the dominance axiom, apply the first result in Lemma 4.6 to the finitely additive measure $\eta$.

\section{Solutions for General Revealed Stochastic Preference Problems.}

5.1. Discussion. Consider the revealed distribution problem of 2.3.1, where $\Pi_{q}$ is a probability on $\mathfrak{X}$ for $\mathrm{q} \in \mathbf{Q}$, and one seeks a probability $\zeta$ on $\mathscr{H}$, or alternately a probability $v$ on $\mathcal{U}$, that rationalizes the observed choice probabilities. Theorem 5.2 establishes that the Axiom of Revealed Stochastic Preference (ARSP) in 2.4 is necessary and sufficient for the existence of a finitely additive probability solving the revealed distribution problem. Its corollaries extend this result to solve the revealed dominating distribution problem. Theorem 5.3 gives regularity conditions under which ARSP is necessary and sufficient for the existence of a countably additive representation solving the revealed distribution problem. Its corollaries show that these regularity conditions are met for a formulation of the classical economic consumer revealed preference problem.

5.2. Theorem. Suppose $\Pi_{q}$ is a finitely additive probability on $\mathscr{X}, \mathrm{q} \in \mathbf{Q}$, satisfying $\Pi_{\mathrm{q}}(\mathbf{C})+\Pi_{\mathrm{q}}\left(\mathbf{C}^{c}\right)$ $=1$ for each $\mathbf{C} \in \mathfrak{X}$. Then ARSP is necessary and sufficient for the existence of a finitely additive probability $\eta$ on $\mathscr{F}$ solving the revealed distribution problem. 
Proof: Recall that $(\mathbf{H}, \mathscr{F})$ is the measurable space of hypothesized decision rules, with $\mathbf{H}(\mathrm{q}, \mathbf{C}) \equiv$ $\{\mathbf{d} \in \mathbf{H} \mid \mathbf{d}(\mathrm{q}) \subseteq \mathbf{C}\}$ for $\mathrm{q} \in \mathbf{Q}$ and $\mathbf{C} \in \mathfrak{X}$. Define the class of sets $\delta=\{\mathbf{H}(\mathrm{q}, \mathbf{C}) \mid \mathrm{q} \in \mathbf{Q}$ and $\mathbf{C} \in \mathfrak{X}\}$. By assumption, $\mathscr{H}$ contains $\sqrt{ }$.

Necessity. Suppose $\eta$ is a finitely additive probability satisfying $\Pi_{\mathrm{q}}(\mathbf{C})=\eta(\mathbf{H}(\mathrm{q}, \mathbf{C}))$ for $\mathrm{q} \in \mathbf{Q}$ and $\mathbf{C}$ $\in \mathfrak{X}$. For a finite sequence of trials $\mathbf{t}=<\left(\mathrm{q}_{1}, \mathbf{C}_{1}\right), \ldots,\left(\mathrm{q}_{\mathrm{m}}, \mathbf{C}_{\mathrm{m}}\right)>$ with $\mathbf{C}_{\mathrm{i}} \in \mathbf{X}, \mathrm{q}_{\mathrm{i}} \in \mathbf{Q}$, define $\mathbf{S}_{\mathrm{i}}=\mathbf{H}\left(\mathrm{q}_{\mathrm{i}}, \mathbf{C}_{\mathrm{i}}\right), \mathrm{i}$ $=1, \ldots, \mathrm{m}$, and $\mathrm{P}\left(\mathbf{S}_{\mathrm{i}}\right)=\eta\left(\mathbf{H}\left(\mathrm{q}_{\mathrm{i}}, \mathbf{C}_{\mathrm{i}}\right)\right)$. Theorem 4.3 then implies that $\eta$ satisfies the dominance axiom; i.e., $\sum_{i=1}^{m} \Pi_{q_{i}}\left(\boldsymbol{C}_{i}\right) \leq \boldsymbol{\alpha}_{\mathbf{H}}(\mathbf{t})$. This condition coincides with ARSP.

Sufficiency. Suppose ARSP. If $\Pi_{q_{1}}\left(\boldsymbol{C}_{1}\right) \geq \Pi_{q_{2}}\left(\boldsymbol{C}_{2}\right)$ and $\mathbf{H}\left(\mathrm{q}_{1}, \mathbf{C}_{1}\right)=\mathbf{H}\left(\mathrm{q}_{2}, \mathbf{C}_{2}\right)$, then

$$
\Pi_{q_{1}}\left(\boldsymbol{C}_{1}\right)+\Pi_{q_{s}}\left(\boldsymbol{C}_{2}{ }^{c}\right) \leq \alpha_{\mathbf{H}}\left(<\mathbf{H}\left(\mathrm{q}_{1}, \mathbf{C}_{1}\right), \mathbf{H}\left(\mathrm{q}_{2}, \mathbf{C}_{2}{ }^{c}\right)>\right)=1,
$$

implying $\Pi_{q_{1}}\left(\boldsymbol{C}_{1}\right) \leq \Pi_{q_{2}}\left(\boldsymbol{C}_{2}\right)$. Hence, one can define uniquely a set function P on $\delta$ satisfying $\mathrm{P}(\mathbf{H}(\mathrm{q}, \mathbf{C}))=\Pi_{\mathrm{q}}(\mathbf{C}), \mathbf{C} \in \mathfrak{X}, \mathrm{q} \in \mathbf{Q}$. By construction, $\mathrm{P}$ satisfies the dominance axiom. Theorem 4.3 then establishes that the dominance problem has a solution, and hence that there exists a finitely additive probability $\eta$ on $\mathbf{H}$ such that $\Pi_{\mathrm{q}}(\mathbf{C}) \leq \eta(\mathbf{H}(\mathrm{q}, \mathbf{C}))$ for $\mathbf{C} \in \mathfrak{X}, \mathbf{q} \in \mathbf{Q}$. Since $\Pi_{\mathrm{q}}$ is a probability satisfying $\Pi_{\mathrm{q}}(\mathbf{C})+\Pi_{\mathrm{q}}\left(\mathbf{C}^{\mathrm{c}}\right)=1$, this solution satisfies $\Pi_{\mathrm{q}}(\mathbf{C})=\eta(\mathbf{H}(\mathrm{q}, \mathbf{C}))$ for $\mathbf{C} \in \mathfrak{X}, \mathrm{q} \in \mathbf{Q}$, and hence solves the revealed distribution problem.

5.2.1. Corollary to Theorem 5.2. If $\Pi_{\mathrm{q}}$ is a non-negative bounded set function on $\mathfrak{X}, \mathrm{q} \in \mathbf{Q}$, then a necessary and sufficient condition for the existence of a finitely additive probability $\eta$ on $\mathscr{H}$ satisfying $\Pi_{\mathrm{q}}(\mathbf{C}) \leq \eta(\mathbf{H}(\mathrm{q}, \mathbf{C}))$ for $\mathbf{C} \in \mathfrak{X}, \mathrm{q} \in \mathbf{Q}$, is that $\Pi_{\mathrm{q}}$ satisfy ARSP.

5.2.2. Corollary to Theorem 5.2. If $\Pi_{q}{ }^{-}$and $\Pi_{q}^{+}$are non-negative bounded set functions on $\mathfrak{X}, \mathrm{q} \in$ $\mathbf{Q}$, then a necessary and sufficient condition for the existence of a finitely additive probability $\eta$ on $\mathscr{K}$ solving the revealed dominating distribution problem is that the function $\Pi_{\mathrm{q}}$ on $\mathfrak{X}, \mathrm{q} \in \mathbf{Q}$ defined by $\Pi_{\mathrm{q}}(\mathbf{C})$ $=\max \left\{\Pi_{\mathrm{q}}(\mathbf{C}), 1-\Pi_{\mathrm{q}}^{+}\left(\mathbf{C}^{\mathrm{c}}\right)\right\}$ satisfy ARSP. 
Proof: Necessity of ARSP. If there exists a probability $\eta$ on $\mathscr{H}$ such that $\Pi_{\mathrm{q}}^{-}(\mathbf{C}) \leq \eta(\mathbf{H}(\mathrm{q}, \mathbf{C})) \leq \Pi^{+}(\mathbf{C})$ for all $\mathbf{C} \in \mathfrak{X}$, then $\eta\left(\mathbf{H}\left(\mathrm{q}, \mathbf{C}^{\mathrm{c}}\right)\right) \leq \Pi_{\mathrm{q}}^{+}\left(\mathbf{C}^{\mathrm{c}}\right)$, implying $1-\Pi^{+}{ }_{\mathrm{q}}\left(\mathbf{C}^{\mathrm{c}}\right) \leq \eta(\mathbf{H}(\mathrm{q}, \mathbf{C}))$, and hence $\eta\left(\mathbf{H}\left(\mathrm{q}, \mathbf{C}^{\mathrm{c}}\right)\right) \geq$ $\Pi_{\mathrm{q}}(\mathbf{C})$. Corollary 5.2.2 then implies that $\Pi_{\mathrm{q}}$ satisfies ARSP.

Sufficiency of ARSP. If $\Pi_{\mathrm{q}}$ satisfies ARSP, then by Corollary 5.2.1, there exists $\eta$ on $\mathscr{H}$ such that $\Pi_{\mathrm{q}}(\mathbf{C})$ $\leq \eta(\mathbf{H}(\mathrm{q}, \mathbf{C}))$. Then $\Pi_{\mathrm{q}}^{-}(\mathbf{C}) \leq \eta(\mathbf{H}(\mathrm{q}, \mathbf{C}))$ and $1-\Pi_{\mathrm{q}^{+}}\left(\mathbf{C}^{\mathrm{c}}\right) \leq \eta\left(\mathbf{H}\left(\mathrm{q}, \mathbf{C}^{\mathrm{c}}\right)\right)$ imply the result.

5.3. Theorem. Suppose the universe of alternatives $\mathbf{X}$ is a complete separable metric space, and let $\mathfrak{X}$ be its Borel $\sigma$-field. Suppose the feasible choice sets $\mathbf{B}(q)$ are non-empty compact subsets of $\mathbf{X}$. Suppose the set $\mathbf{H}$ of decision rules consistent with a hypothesis of rationality is given a topology whose basis are the sets $\mathbf{H}(\mathrm{q}, \mathbf{C})$ for $\mathrm{q} \in \mathbf{Q}$ and open $\mathbf{C} \in \mathfrak{X}$. Suppose that $\mathbf{H}$ is a compact space in this topology, and let $\mathscr{H}$ be its Borel $\sigma$-field. Suppose $\Pi_{\mathrm{q}}$ is a countably additive probability on $\mathfrak{X}, \mathrm{q} \in \mathbf{Q}$, satisfying $\Pi_{\mathrm{q}}(\mathbf{C})+\Pi_{\mathrm{q}}\left(\mathbf{C}^{\mathrm{c}}\right)=1$ for each $\mathbf{C} \in \mathfrak{X}$, and $\Pi_{\mathrm{q}}(\mathbf{B}(\mathrm{q}))=1$. Then ARSP is necessary and sufficient for the existence of a countably additive probability $\eta$ on $\mathscr{H}$ solving the revealed distribution problem.

Proof: The necessity of ARSP is immediate from Theorem 5.2. To prove sufficiency, suppose ARSP holds, and that $\eta$ is a finitely additive probability, given by Theorem 5.2, that satisfies $\eta(\mathbf{H}(\mathrm{q}, \mathbf{C}))=\Pi_{\mathrm{q}}(\mathbf{C})$ for $\mathrm{q} \in \mathbf{Q}, \mathbf{C} \in \mathbf{X}$. For $\mathbf{C} \in \mathfrak{X}$ open, the set $\mathbf{H}(\mathrm{q}, \mathbf{C})^{\mathrm{c}}$ is closed by construction, and satisfies $\eta\left(\mathbf{H}(\mathrm{q}, \mathbf{C})^{\mathrm{c}}\right)=$ $1-\Pi_{\mathrm{q}}(\mathbf{C})=\Pi_{\mathrm{q}}\left(\mathbf{C}^{\mathrm{c}}\right)$. Then, the family $\varsigma_{0}=\left\{\mathbf{H}(\mathrm{q}, \mathbf{C})^{\mathrm{c}} \mid \mathrm{q} \in \mathbf{Q}\right.$, open $\left.\mathbf{C} \in \mathfrak{X}\right\}$ is a family of closed subsets of a compact space, and is therefore a compact class. On the family $\varsigma=\left\{\mathbf{H}(\mathrm{q}, \mathbf{C})^{\mathrm{c}} \mid \mathrm{q} \in \mathbf{Q}, \mathbf{C} \in \mathcal{X}\right\}, \eta$ satisfies

$$
\left.\eta\left(\mathbf{H}(\mathrm{q}, \mathbf{C})^{\mathrm{c}}\right)=\Pi_{\mathrm{q}}\left(\mathbf{C}^{\mathrm{c}}\right)=\sup \left\{\Pi_{\mathrm{q}}\left(\mathbf{C}^{\prime c}\right)\right) \mid \mathbf{C}^{\prime} \text { open, } \mathbf{C} \subseteq \mathbf{C}^{\prime}\right\}=\sup \left\{\eta\left(\mathbf{H}\left(\mathrm{q}, \mathbf{C}^{\prime}\right)^{\mathrm{c}}\right) \mid \mathbf{C}^{\prime} \text { open, } \mathbf{C} \subseteq \mathbf{C}^{\prime}\right\},
$$

since by Lemma $4.6 \Pi_{q}$ is countably additive, hence tight, on the compact feasible choice sets $\mathbf{B}(q)$. Therefore, $\eta$ is tight on $\varsigma$, and Lemma 4.6 implies that it is countably additive on $\mathcal{H}$.

5.3.1. Corollary. Suppose $\mathbf{X}$ is a convex compact metric space with metric $\rho$, and the feasible choice situations $\mathbf{B}(q)$ are convex closed non-empty subsets of $\mathbf{X}$. Suppose $\Pi_{q}$ is a (countably additive) probability on $\mathfrak{X}, \mathrm{q} \in \mathbf{Q}$, satisfying $\Pi_{\mathrm{q}}(\mathbf{C})+\Pi_{\mathrm{q}}\left(\mathbf{C}^{\mathrm{c}}\right)=1$ for each $\mathbf{C} \in \mathfrak{X}$, and $\Pi_{\mathrm{q}}(\mathbf{B}(\mathrm{q}))=1$. Suppose decision-makers are hypothesized to maximize utilities from a family $\mathbf{U}$ of uniformly bounded functions on $\mathbf{X}$ that are equicontinuous; i.e., for each $\varepsilon>0$ there exists $\delta>0$ such that $\mathrm{x}, \mathrm{x}^{\prime} \in \mathbf{X}$ and $\rho\left(\mathrm{x}, \mathrm{x}^{\prime}\right)<\delta$ 
implies $\sup _{\mathrm{u} \in \mathrm{U}}\left|\mathrm{u}(\mathrm{x})-\mathrm{u}\left(\mathrm{x}^{\prime}\right)\right|<\varepsilon$. Then ARSP is necessary and sufficient for the existence of a (countably additive) probability $v$ on $U$ solving the revealed distribution problem.

Proof: The Arzela-Ascoli theorem (Dunford and Schwartz, 1964, IV.6.8) establishes that U is a compact subset of the space $\mathbf{C}(\mathbf{X})$ of continuous functions on $\mathbf{X}$, with $\|\mathbf{u}\|=\sup _{\mathrm{x} \in \mathbf{X}}|\mathbf{u}(\mathrm{x})|$ for $\mathbf{u} \in \mathbf{C}(\mathbf{X})$. For open $\mathbf{C} \in \mathfrak{X}$, the set $\mathbf{U}(\mathrm{q}, \mathbf{C})=\left\{\mathrm{u} \in \mathbf{U} \mid \sup _{\mathrm{x} \in \mathbf{C} n \mathbf{B}(q)} \mathrm{u}(\mathrm{x})>\sup _{\left.\mathrm{x}^{\prime} \in \mathbf{B}(q)\right) \mathbf{C}} \mathbf{u}\left(\mathrm{x}^{\prime}\right)\right\}$ is open. To show this, suppose $\mathrm{u} \in$ $\mathbf{U}(\mathrm{q}, \mathbf{C})$. Then there exists $\mathrm{x}^{\prime \prime} \in \mathbf{B}(\mathrm{q})$ with $\mathrm{u}\left(\mathrm{x}^{\prime \prime}\right)>\sup _{\mathrm{x}^{\prime} \in \mathbf{B}(\mathrm{q}) \mathbf{C}} \mathrm{u}\left(\mathrm{x}^{\prime}\right)+\varepsilon$ for some $\varepsilon>0$. Consider $\mathrm{u}^{\prime}$ satisfying $\left\|\mathrm{u}-\mathrm{u}^{\prime}\right\|<\varepsilon / 3$. Then, $\mathrm{u}^{\prime}\left(\mathrm{x}^{\prime \prime}\right)>\sup _{\left.\mathrm{x}^{\prime} \in \mathbf{B}(\mathrm{q})\right) \mathrm{c}^{\prime}} \mathrm{u}^{\prime}\left(\mathrm{x}^{\prime}\right)+\varepsilon / 3$, implying $\mathrm{u}^{\prime} \in \mathbf{U}(\mathrm{q}, \mathbf{C})$. Hence, $\mathbf{U}(\mathrm{q}, \mathbf{C})$ with $\mathbf{C}$ open is an open set in $\mathbf{U}$. Theorem 5.3 then gives the result.

5.3.2. Corollary. Suppose $\mathbf{X}$ is a convex compact subset of a locally convex normed linear space $\mathbf{L}$, and the feasible choice situations $\mathbf{B}(q)$ are convex closed non-empty subsets of $\mathbf{X}$. Suppose $\Pi_{q}$ is a countably additive probability on $\mathfrak{X}$ for $\mathrm{q} \in \mathbf{Q}$, satisfying $\Pi_{\mathrm{q}}(\mathbf{C})+\Pi_{\mathrm{q}}\left(\mathbf{C}^{\mathrm{c}}\right)=1$ for each $\mathbf{C} \in \mathfrak{X}$, and $\Pi_{\mathrm{q}}(\mathbf{B}(\mathrm{q}))=1$. Suppose decision-makers are hypothesized to maximize utilities from a family $\mathbf{U}$ of uniformly bounded and concave functions on an open set containing $\mathbf{X}$. Then ARSP is necessary and sufficient for the existence of a countably additive probability $v$ on $\mathcal{U}$ solving the revealed distribution problem.

Proof: Assume that the uniform bound on $\mathbf{u} \in \mathbf{U}$ is $\|\mathbf{u}\| \leq 1$. Each point in $\mathbf{X}$ has an open neighborhood that is contained in the open set on which utility functions are defined. Since $\mathbf{X}$ is compact, it has a maximum diameter $\mu$. Also, one can extract from the open neighborhoods a finite sub-cover; let $\lambda$ be the diameter of the smallest neighborhood in this sub-cover. Suppose $\mathrm{x}, \mathrm{x}^{\prime} \in \mathbf{X}$. By construction, the domain of the functions in $\mathbf{U}$ contains $\mathrm{x}-\left(\mathrm{x}^{\prime}-\mathrm{x}\right) \lambda / \mu$. Then, for $0<\theta<1$, concavity implies

$$
u\left((1-\theta) x+\theta x^{\prime}\right) \geq(1-\theta) u(x)+\theta u\left(x^{\prime}\right), \text { or } u\left(x+\theta\left(x^{\prime}-x\right)\right)-u(x) \geq \theta\left[u\left(x^{\prime}\right)-u(x)\right] \geq-2 \theta
$$

and

$$
\begin{gathered}
\mathrm{u}(\mathrm{x})=\mathrm{u}\left((\lambda /(\theta \mu+\lambda))\left(\mathrm{x}+\theta\left(\mathrm{x}^{\prime}-\mathrm{x}\right)\right)+(\theta \mu /(\theta \mu+\lambda))\left(\mathrm{x}-\left(\mathrm{x}^{\prime}-\mathrm{x}\right) \lambda / \mu\right)\right) \\
\geq(\lambda /(\theta \mu+\lambda)) \mathrm{u}\left(\mathrm{x}+\theta\left(\mathrm{x}^{\prime}-\mathrm{x}\right)\right)+(\theta \mu /(\theta \mu+\lambda)) \mathrm{u}\left(\mathrm{x}-\left(\mathrm{x}^{\prime}-\mathrm{x}\right) \lambda / \mu\right), \text { or } \\
\mathrm{u}\left(\mathrm{x}+\theta\left(\mathrm{x}^{\prime}-\mathrm{x}\right)\right)-\mathrm{u}(\mathrm{x}) \leq(\theta \mu /(\theta \mu+\lambda))\left[\mathrm{u}\left(\mathrm{x}+\theta\left(\mathrm{x}^{\prime}-\mathrm{x}\right)\right)-\mathrm{u}\left(\mathrm{x}-\left(\mathrm{x}^{\prime}-\mathrm{x}\right) \lambda / \mu\right)\right] \leq 2 \theta \mu / \lambda .
\end{gathered}
$$

Given $\varepsilon>0$, choose $\theta=\varepsilon \cdot \min (1 / 2, \lambda / 2 \mu)$. Then, $\mathbf{U}$ satisfies the condition that $\mathrm{x}, \mathrm{x}^{\prime} \in \mathbf{X}$ with $\rho\left(\mathrm{x}, \mathrm{x}^{\prime}\right)<$ $\theta$ implies $\left|\mathrm{u}(\mathrm{x})-\mathrm{u}\left(\mathrm{x}^{\prime}\right)\right|<\varepsilon$ for all $\mathrm{u} \in \mathbf{U}$, and Corollary 5.3.1 gives the result. 
5.4. Remarks. Theorem 5.3 is difficult to apply without sufficient conditions for the compactness of the set $\mathbf{H}$ of hypothesized decision rules. Corollary 5.3.2, which was suggested by Rosa Matzkin, provides conditions which correspond to the classical revealed preference problem. The requirement that the utility functions $\mathrm{u} \in \mathbf{U}$ be defined on an open set containing $\mathbf{X}$ can be replaced by a condition on the subgradient $\Gamma(\mathrm{x}, \mathrm{u})=\left\{\mathrm{p} \in \mathrm{L}^{*} \mid \mathrm{u}(\mathrm{y})-\mathrm{u}(\mathrm{x}) \leq \mathrm{p}(\mathrm{y}-\mathrm{x})\right.$ for $\left.\mathrm{y} \in \mathrm{X}\right\}$ that there exist a bound $\mathrm{K}>0$ such that $\varnothing \neq$ $\Gamma(\mathrm{x}, \mathrm{u}) \cap\left\{\mathrm{p} \in \mathrm{L}^{*} \mid\|\mathrm{p}\|<\mathrm{K}\right\}$ for $\mathrm{x} \in \mathrm{X}, \mathrm{u} \in \mathrm{U}$; see Matzkin (1992), Brown and Matzkin (1996).

If in Corollary 5.3.1, $\mathbf{Q}$ is compact and $\mathbf{B}(q)$ is a continuous correspondence, then it is sufficient to test ARSP for trial sequences drawn from a countable subset of the set of possible trials, and if ARSP fails, this will be detected in a finite number of trials; see McFadden (1979). Thus, under these regularity conditions, a test of the validity of ARSP is computable. Going further, one can consider a net formed by nests of trial sequences $\mathbf{t}=<\left(\mathrm{q}_{1}, \mathrm{C}_{1}\right), \ldots,\left(\mathrm{q}_{\mathrm{m}}, \mathrm{C}_{\mathrm{m}}\right)>$; i.e., sequences $\mathbf{t}_{1} \subseteq \mathbf{t}_{2} \subseteq \ldots$, and utilize the linear program in Theorem 3.1 (c) to recover the convex closed sets $\mathbf{G}_{\mathbf{t}}$ of rationalizing probabilities on the finite algebras of subsets of $\mathbf{H}$ induced by the trial sequences $\mathbf{t}_{\mathrm{k}}$, provided ARSP holds. For each set $\mathbf{H}_{1}$ in the Boolean algebra generated by the $\mathbf{H}(\mathrm{q}, \mathbf{C})^{\mathrm{c}}$ for $\mathbf{C}$ open and $\mathrm{q} \in \mathbf{Q}$, the net formed by the probabilities $\eta_{\mathbf{t}}\left(\mathbf{H}_{1}\right)$ for $\eta_{\mathbf{t}} \in \mathbf{G}_{\mathbf{t}}$ and a net of trial sequences $\mathbf{t}$ containing the trials that enter the finite intersection and union operations that produce $\mathbf{H}_{1}$ will contain a sub-net that converges to $\eta\left(\mathbf{H}_{1}\right)$ for a probability $\eta$ that solves the revealed distribution problem in Theorem 5.2. Thus, there is a sequence of finite linear programming problems that provide a computable test of ARSP, and computable bounds for the rationalizing probabilities.

Two published papers have considered somewhat different versions of the issue of countably additive rationalizations. McFadden (1975) examines the question of when a joint probability over endowments and a compact set of preferences can be found that rationalize observed moments, such as per capita mean market demands. By restricting and redefining the observed moments, the general moment problem can be specialized to the revealed distribution problem. Cohen (1980) extends the finite analysis considered in Section 3 to the case where $\mathbf{X}$ is infinite, but all choice sets $\mathbf{B}(q), q \in \mathbf{Q}$, are finite. The Block-Marschak polynomials are defined for each finite restriction of $\mathbf{X}$, and a net of choice sets contained in this restriction. Now consider a net of nested restrictions of $\mathbf{X}$, and generalized sequences of the probability measures constructed by Falmagne's method, as described in Theorem 3.3. Conditions are then given under which a generalized subsequence has a countably additive limit. Cohen's proof is 
difficult, but the essential idea is that when choices can be rationalized for all nested sequences of finite $\mathbf{X}$, and compactness conditions hold in the limit so that there can be no countable union of disjoint sets with positive measure, then the Kolmogorov consistency theorem and the Caratheodory extension theorem apply to achieve countable additivity. Theorem 5.3 and its corollaries provide more easily checked conditions for countable additivity, and handle the economic choice application where choice sets are not finite.

\section{Extensions}

New revealed preference problems can be generated by varying the family of feasible choice sets, the class of permissible decision rules, and the structure of observations. For example, one could consider classes of permissible choice rules that are either more restrictive than classical preference maximization (e.g., optimization of smooth preferences, or preferences that are homothetic, have linear Engle curves, or are in parametric families) or less restrictive (e.g., incomplete optimization of preferences, preferences that are not preorders, or preferences that are context or perception-dependent). ${ }^{5}$ One could also consider observational situations encountered in practice (e.g., composition of market and experimental choice data, conditional distributions or conditional moments of choices given observable consumer characteristics). The classical revealed preference problem is traditionally formulated under the assumption that an individual's choices are observed in a sequence of static budget situations without carry-over of durables, experience, or learning from one situation to the next. The revealed distribution problem assumes that individuals are not tracked and that information is collected only on a population's distributions of choices. However, our analysis of this problem has maintained the assumption that the budget situations are static, without dynamics introduced by intertemporal maximization and state dependence. A much broader class of revealed preference problems could be formulated that allow these dynamic elements, and account explicitly for the panel data structure implicit in observation of repeated choice situations. For example, the observed choices of an individual in repeated choice situations may be interpreted as a realization of a stochastic process indexed by the choice situations, and the distribution

\footnotetext{
${ }^{5}$ Homotheticity restrictions permit stochastic preference versions of the computational tests of revealed preference theory developed by Varian (1982,1983). One could go further and formulate parametric or nonparametric econometric tests of ARSP for a variety of hypothesized decision models.
} 
of the stochastic process in a population, or its moments, may constitute the observations that can be analyzed.

The Axiom of Revealed Stochastic Preference can be applied to many classes of permissible choice rules; the only modifications come in the properties and interpretation of the choice probabilities and the determination of intersection numbers for trial sequences. For an expanded menu of revealed preference problems, if observed choice data are consistent with the specified class of permissible choice rules, additional interesting questions arise: Do the observations identify a unique distribution, or identify bounds on the possible distributions (McFadden, 1975)? Can the analysis be made conditional on observed population characteristics, with solutions that reflect the systematic variation in choice distributions with these characteristics? Many of these extended revealed stochastic preference problems have not been studied, and deserve the attention of economic theorists.

\section{References}

Bandyopadhyay, T.; I. Dasgupta; P. Pattanaik (1999) "Stochastic Revealed Preference and the Theory of Demand," Journal of Economic Theory, 84, 95-110.

Barbera, S.' P. Pattanaik (1986) "Falmagne and the Rationalizability of Stochastic Choices in Terms of Random Orderings," Econometrica, 54, 707-716.

Barbara, S. (1991) "Rationalizable Stochastic Choice over Restricted Domains," in J. Chipman, D. McFadden, and K. Richter (eds) Preferences, Uncertainty, and Rationality, Westview Press, 203-217.

Block, H.; J. Marschak (1960) "Random orderings and stochastic theories of response," in I. Olkin et al (eds) Contributions to Probability and Statistics. Stanford University Press, 97-132.

Brown, D.; R. Matzkin (1996) “Testable Restrictions on the Equilibrium Manifold,” Econometrica, 64, 1249-1262.

Cohen, M. (1980) "Random Utility Systems -- the Infinite Case," Journal of Mathematical Psychology," 22, 1-23.

Cohen, M.; J. Falmagne (1990) "Random Utility Representation of Binary Choice Probabilities: A New Class of Necessary Conditions," Journal of Mathematical Psychology, 34, 88-94.

Dunford, N.; J. Schwartz (1964) Linear Operators, Interscience.

Falmagne, J. (1978) "A Representation Theorem for Finite Random Scale Systems," Journal of Mathematical Psychology, $18,52-72$.

Debreu, G. (1967) "Preference Functions on a Measure Space of Economic Agents," Econometrica, 35, 111-122.

Fishburn, P. (1978) "Choice Probabilities and Choice functions," Jounal of Mathematical Psychology, 10, $327-352$.

Fishburn, P.; J. Falmagne (1989) "Binary Choice Probabilities and Rankings," Economic Letters, 31, $113-117$.

Fishburn, P. (1992) "Induced Binary Probabilities and the Linear Ordering Polytope: A Status Report," Mathematical Social Sciences, 23, 67-80.

Fishburn, P. (1998) "Stochastic Utility," in S. Barbara, P. Hammond, C. Seidl (eds) Handbook of Utiltiy Theory, Kluwer, 273-320.

Freedman, D.; R. Purves (1969) "Bayes Method for Bookies," Annals of Mathematical Statistics, 40, $117-1186$.

Halldin, C. (1974) "The Choice Axiom, Revealed Preference, and the Theory of Demand," Theory and Decision, 5, 139-160. Hildenbrand, W. (1971) "Random Preferences and Equilibrium Analysis," Journal of Economic Theory, 3, 414-429.

Hildenbrand, W. (1974) Core and Equilibrium of a Large Economy, Princeton University Press.

Houthakker, H. (1950) "Revealed Preference and the Utility Function," Economica, 17, 159-174.

Karlin, S. (1959) Mathematical Methods and Theory in Games, Programming, and Economics, Addison-Wesley, $265-273$. Luce, D. (1959) Individual Choice Behavior, Wiley. 
Luce, D.; P. Suppes (1965) "Preferences, Utility, and Subjective Probability," in D. Luce et al (eds) Handbook of Mathematical Psychology III, Wiley, 249-410.

Manski, C. (1977) "The Structure of Random Utility Models," Theory and Decision, 8, 229-254.

Marschak, J. (1960) "Binary-Choice Constrains on Random Utility Indicators," in K. Arrow, S. Karlin, P. Suppes (eds) Mathematical Methods in the Social Sciences, Stanford University Press, 312-329.

Matzkin, R. (1992) "Nonparametric and Distribution-Free Estimation of the Binary Threshold Crossing and the Binary Choice Models," Econometrica, 60, 239-270.

McCausland, W. (2002) "A Theory of Random Consumer Demand," working paper.

McFadden, D.; M. K. Richter (1971) "On the Extension of a Set Function on a Set of Events to a Probability on the Generated Boolean $\sigma$-Algebra," University of California, Berkeley, working paper.

McFadden, D. (1973) "Estimation and Testing of Models of Individual Choice Behavior from Data on a Population of Subjects," University of California, Berkeley, working paper.

McFadden, D. (1974) "Conditional Logit Analysis of Qualitative Choice Behavior," in P. Zarembka (ed) Frontiers of Econometrics, Academic Press, 105-142.

McFadden, D. (1975) "Tchebyscheff Bounds for the Space of Agent Characteristics," Journal of Mathematical Economics, $2,225-242$.

McFadden, D. (1979) "A Note on the Computability of the Strong Axiom of Revealed Preference," Journal of Mathematical Economics, 6, 15-16.

McFadden, D.; K. Richter (1991) "Stochastic Rationality and Revealed Stochastic Preference," in J. Chipman, D. McFadden, K. Richter (eds) Preferences, Uncertainty, and Rationality, Westview Press, 161-186.

McFadden, D. (2001) "Economic Choices," American Economic Review, 91, 351-378.

McLennan, A. (1991) "Binary Stochastic Choice," in J. Chipman, D. McFadden, and K. Richter (eds) Preferences, Uncertainty, and Rationality, Westview Press, 187-202.

Neveu, J. (1965) Mathematical Foundations of the Calculus of Probability, Holden-Day.

Richter, K. (1966) "Revealed Preference Theory," Econometrica, 34, 635-645.

Richter, K. (1971) "Rational Choice," in J. Chipman et al (eds) Preferences, Uncertainty, and Optimality, Harcourt-Brace.

Samuelson, P. (1938) "A Note on the Pure Theory of Consumer Behavior," Economica, 51- 71 and 353-354.

Thurstone, L. (1927) "A Law of Comparative Judgment," Psychological Review, 34, 273- 286.

Varian, H. (1982) "The Nonparametric Approach to Demand Analysis," Econometrica, 50, 945-974.

Varian, H. (1983) "Nonparametric Tests of Consumer Behavior," Review of Economic Studies, 50, 99-110. 